\title{
A TEST OF EQUALITY OF MEAN VECTORS OF SEVERAL HETEROSCEDASTIC MULTIVARIATE POPULATIONS
}

\author{
Yoshihide Kakizawa*
}

\begin{abstract}
This paper deals with a test of equality of mean vectors of several heteroscedastic multivariate populations. We derive not only the asymptotic expansion up to $N^{-1}$ of the nonnull distribution of James's (1954) statistic, but also those of two corrected statistics due to Cordeiro and Ferrari (1991) and Kakizawa (1996). The derivation we considered here is based on the differential operator method developed in Kakizawa and Iwashita (2005).
\end{abstract}

Key words and phrases: Asymptotic expansion, Bartlett's type adjustment, differential operator, heteroscedasticity, local power, nonnormality, nonnull distribution, one-way MANOVA.

\section{Introduction}

A statistical hypothesis in multivariate analysis is usually tested on the assumption that the observations are independently and normally distributed with a common covariance matrix. A question of theoretical and practical importance is the robustness of inference methods with respect to violation of normality or equality of covariance matrices (see Ito (1969)). Since Kano (1995) and Fujikoshi (1997), there have been many works to examine the effect of nonnormality upon standard multivariate test statistics on a general linear hypothesis of one-way MANOVA model, multivariate linear regression model and GMANOVA model. See Yanagihara (2001), Fujikoshi (2002a, 2002b), Wakaki et al. (2002), Kakizawa and Iwashita (2005, 2008), Kakizawa (2005, 2006) and Gupta et al. (2006) for recent developments in asymptotic expansions of the null or nonnull distributions of some test statistics according to situations under consideration. On the other hand, there is little progress in the multivariate nonnormal heteroscedastic case (see Kakizawa and Iwashita (2005) for the multivariate Behrens-Fisher problem). The present paper is the multivariate extension to Yanagihara (2000), who derived an asymptotic expansion of the null distribution of James's (1954) statistic under heteroscedastic univariate nonnormal populations.

Suppose that $\left\{\boldsymbol{Y}_{1}^{(1)}, \ldots, \boldsymbol{Y}_{N_{1}}^{(1)}\right\}, \ldots,\left\{\boldsymbol{Y}_{1}^{(q)}, \ldots, \boldsymbol{Y}_{N_{q}}^{(q)}\right\}$ are $q$ independent samples from $p$-variate distributions with mean vector $\boldsymbol{\mu}^{(a)}$ and positive definite covariance matrix $\Sigma^{(a)}(a=1, \ldots, q)$, where $N=N_{1}+\cdots+N_{q}$ is the total number of observations. Define the sample mean vector and covariance matrix

\footnotetext{
Received May 30, 2006. Revised August 18, 2006. Accepted September 10, 2006.

*Faculty of Economics, Hokkaido University, Nishi 7, Kita 9, Kita-ku, Sapporo 060-0809, Japan. Email: kakizawa@econ.hokudai.ac.jp
} 
of $\boldsymbol{Y}_{1}^{(a)}, \ldots, \boldsymbol{Y}_{N_{a}}^{(a)}$ by

$$
\overline{\boldsymbol{Y}}^{(a)}=\frac{1}{N_{a}} \sum_{i=1}^{N_{a}} \boldsymbol{Y}_{i}^{(a)} \quad \text { and } \quad S_{Y}^{(a)}=\frac{1}{N_{a}-1} \sum_{i=1}^{N_{a}}\left(\boldsymbol{Y}_{i}^{(a)}-\overline{\boldsymbol{Y}}^{(a)}\right)\left(\boldsymbol{Y}_{i}^{(a)}-\overline{\boldsymbol{Y}}^{(a)}\right)^{\prime} .
$$

For testing $H: \boldsymbol{\mu}^{(1)}=\cdots=\boldsymbol{\mu}^{(q)}$ vs $A: \boldsymbol{\mu}^{(a)} \neq \boldsymbol{\mu}^{\left(a^{\prime}\right)}$ for some $a, a^{\prime} \in\{1, \ldots, q\}$, the following test statistic was proposed by James $(1954,(7 \cdot 18))$ under normality:

$$
T_{J}^{2}=N \sum_{a=1}^{q}\left(\overline{\boldsymbol{Y}}^{(a)}-\widehat{\boldsymbol{Y}}\right)^{\prime} W_{Y}^{(a)}\left(\overline{\boldsymbol{Y}}^{(a)}-\widehat{\overline{\boldsymbol{Y}}}\right)
$$

where

$$
\begin{aligned}
& \rho_{a}=\frac{N_{a}^{1 / 2}}{N^{1 / 2}}, \quad W_{Y}^{(a)}=\rho_{a}^{2}\left(S_{Y}^{(a)}\right)^{-1} \\
& W_{Y}=\sum_{a=1}^{q} W_{Y}^{(a)}, \quad \widehat{\boldsymbol{Y}}=W_{Y}^{-1} \sum_{a=1}^{q} W_{Y}^{(a)} \overline{\boldsymbol{Y}}^{(a)} .
\end{aligned}
$$

His test statistic can be explained as follows. If covariance matrices $\Sigma^{(1)}, \ldots, \Sigma^{(q)}$ are known, then the likelihood ratio criterion under assumptions of multivariate normality becomes

$$
N \sum_{a=1}^{q}\left(\overline{\boldsymbol{Y}}^{(a)}-\widehat{\boldsymbol{\mu}}_{0}\right)^{\prime} \Lambda^{(a)}\left(\overline{\boldsymbol{Y}}^{(a)}-\widehat{\boldsymbol{\mu}}_{0}\right),
$$

where

$$
\Lambda^{(a)}=\rho_{a}^{2}\left(\Sigma^{(a)}\right)^{-1}, \quad \Lambda=\sum_{a=1}^{q} \Lambda^{(a)}, \quad \widehat{\boldsymbol{\mu}}_{0}=\Lambda^{-1} \sum_{a=1}^{q} \Lambda^{(a)} \overline{\boldsymbol{Y}}^{(a)} .
$$

The feasible statistic $T_{J}^{2}$ is given by replacing unknown covariance matrices $\Sigma^{(1)}, \ldots, \Sigma^{(q)}$ by their unbiased estimators $S_{Y}^{(1)}, \ldots, S_{Y}^{(q)}$. James $(1954,(7 \cdot 3)$ $(7 \cdot 5)$ and $(7 \cdot 18))$ also noted that $T_{J}^{2}$ can be written as

$$
T_{J}^{2}=\left(N^{1 / 2} \overline{\boldsymbol{Y}}^{*}\right)^{\prime} \boldsymbol{V}_{Y}^{-1}\left(N^{1 / 2} \overline{\boldsymbol{Y}}^{*}\right)
$$

where

$$
\overline{\boldsymbol{Y}}^{*}=\left(\begin{array}{c}
\overline{\boldsymbol{Y}}^{(1)}-\overline{\boldsymbol{Y}}^{(q)} \\
\vdots \\
\overline{\boldsymbol{Y}}^{(q-1)}-\overline{\boldsymbol{Y}}^{(q)}
\end{array}\right)
$$

and

$$
\boldsymbol{V}_{Y}^{-1}=\operatorname{diag}\left(W_{Y}^{(1)}, \ldots, W_{Y}^{(q-1)}\right)
$$




$$
-\operatorname{diag}\left(W_{Y}^{(1)}, \ldots, W_{Y}^{(q-1)}\right)\left\{\left(\mathbf{1}_{q-1} \mathbf{1}_{q-1}^{\prime}\right) \otimes W_{Y}^{-1}\right\} \operatorname{diag}\left(W_{Y}^{(1)}, \ldots, W_{Y}^{(q-1)}\right),
$$

with $\mathbf{1}_{q-1}=\overbrace{(1, \ldots, 1)^{\prime}}^{(q-1) \text { times }}$ Here, $\operatorname{diag}\left(W_{Y}^{(1)}, \ldots, W_{Y}^{(q-1)}\right)$ denotes the block diagonal matrix whose $b$-th diagonal block is $W_{Y}^{(b)}$. The purpose of this paper is not only to derive the asymptotic expansion of the nonnull distribution $\operatorname{Pr}\left(T_{J}^{2} \leq x\right)$ for $x>0$, but also to consider two corrected statistics due to Cordeiro and Ferrari (1991) and Kakizawa (1996). The derivation we considered here is based on the differential operator method developed in Kakizawa and Iwashita $(2005,2008)$ and Kakizawa $(2005,2006)$ under general distributions.

We end this section by giving some comments on the problem of testing the hypothesis $\boldsymbol{C}^{\prime} \boldsymbol{\mu}^{(1)}=\cdots=\boldsymbol{C}^{\prime} \boldsymbol{\mu}^{(q)}$, where $\boldsymbol{C}^{\prime}$ is an $s \times p$ known matrix of rank $s(\leq p)$. No special treatment for this problem is needed since it reduces to the hypothesis $\widetilde{\boldsymbol{\mu}}^{(1)}=\cdots=\widetilde{\boldsymbol{\mu}}^{(q)}$ on the transformed data $\widetilde{\boldsymbol{Y}}_{i}^{(a)}=\boldsymbol{C}^{\prime} \boldsymbol{Y}_{i}^{(a)}$, where $\widetilde{\boldsymbol{\mu}}^{(a)}=\boldsymbol{C}^{\prime} \boldsymbol{\mu}^{(a)}$. In that case $\widetilde{\boldsymbol{Y}}_{i}^{(a)}-\widetilde{\boldsymbol{\mu}}^{(a)}$ is independently distributed with mean vector $\mathbf{0} \in \boldsymbol{R}^{s}$ and positive definite covariance matrix $\Sigma_{\mathrm{C}}^{(a)} \equiv \boldsymbol{C}^{\prime} \Sigma^{(a)} \boldsymbol{C}$, provided that $\Sigma^{(a)}$ is positive definite (it is easy to see that $\operatorname{rank}\left(\Sigma_{\mathrm{C}}^{(a)}\right)=\operatorname{rank}\left(\boldsymbol{L}_{\Sigma^{(a)}}^{\prime} \boldsymbol{C}\right)=$ $\operatorname{rank}(\boldsymbol{C})=s$, where $\boldsymbol{L}_{\Sigma^{(a)}}$ is the lower triangular matrix with positive diagonal elements satisfying $\left.\boldsymbol{L}_{\Sigma^{(a)}} \boldsymbol{L}_{\Sigma^{(a)}}^{\prime}=\Sigma^{(a)}\right)$.

\section{Nonnull distribution of $T_{J}^{2}$}

Let $\left\{\boldsymbol{Y}_{1}^{(1)}, \ldots, \boldsymbol{Y}_{N_{1}}^{(1)}\right\}, \ldots,\left\{\boldsymbol{Y}_{1}^{(q)}, \ldots, \boldsymbol{Y}_{N_{q}}^{(q)}\right\}$ be $q$ independent samples from population distributions with mean vector $\boldsymbol{\mu}^{(a)}$ and positive definite covariance matrix $\Sigma^{(a)}(a=1, \ldots, q)$, where $q \geq 2$ is a given integer. In other words, the model considered is a multivariate one-way classification model

$$
\boldsymbol{Y}_{i}^{(a)}=\boldsymbol{\mu}^{(a)}+\boldsymbol{U}_{i}^{(a)} \quad\left(a=1, \ldots, q ; i=1, \ldots, N_{a}\right) .
$$

We assume that the $\boldsymbol{U}_{i}^{(a)}$, s are independently distributed according to a common $p$-variate distribution of $\boldsymbol{U}^{(a)}=\left(U_{1}^{(a)}, \ldots, U_{p}^{(a)}\right)^{\prime}$ with mean vector $\mathbf{0}$, positive definite covariance matrix $\Sigma^{(a)}$ and $v$-th order cumulant $\operatorname{Cum}\left(U_{j_{1}}^{(a)}, \ldots, U_{j_{v}}^{(a)}\right)=$ $\kappa_{j_{1}, \ldots, j_{v}}^{(a)}(v \geq 3)$. Here and subsequently we use $j, k$, without or with suffixes, to denote indices, each such index running from 1 to $p$ unless explicitly stated otherwise. We always use $a$, without or with suffixes, to denote indices, each such index running from 1 to $q$. Further, we use $b$, without or with suffixes, to denote indices, each such index running from 1 to $q-1$. Let

$$
\overline{\boldsymbol{U}}^{(a)}=\frac{1}{N_{a}} \sum_{i=1}^{N_{a}} \boldsymbol{U}_{i}^{(a)} \quad \text { and } \quad S_{U}^{(a)}=\frac{1}{N_{a}-1} \sum_{i=1}^{N_{a}}\left(\boldsymbol{U}_{i}^{(a)}-\overline{\boldsymbol{U}}^{(a)}\right)\left(\boldsymbol{U}_{i}^{(a)}-\overline{\boldsymbol{U}}^{(a)}\right)^{\prime} .
$$

Remark 1. For each $a=1, \ldots, q$, the sample covariance matrix $S_{Y}^{(a)}=S_{U}^{(a)}$ is positive definite with probability one if $N_{a}-1 \geq p$, provided that under each 
distribution of $\boldsymbol{U}^{(a)} \in \boldsymbol{R}^{p}$, every flat of dimension $p-1$ has probability zero (see Eaton and Perlman (1973)). However, such a non-asymptotic result can be replaced by a higher-order one, as in (4.1) below.

\section{1. $\quad$ Limiting distribution}

Let

$$
W_{U}^{(a)}=\rho_{a}^{2}\left(S_{U}^{(a)}\right)^{-1}, \quad W_{U}=\sum_{a=1}^{q} W_{U}^{(a)} .
$$

Under a local alternative

$$
A_{N}: \boldsymbol{\mu}^{(a)}=\boldsymbol{\mu}+\frac{\varepsilon^{(a)}}{N^{1 / 2}} \quad(a=1, \ldots, q),
$$

we can write James's (1954) statistic as

$$
T_{J}^{2}=\left(N^{1 / 2} \overline{\boldsymbol{Y}}^{*}\right)^{\prime} \boldsymbol{V}_{Y}^{-1}\left(N^{1 / 2} \overline{\boldsymbol{Y}}^{*}\right)=\left(N^{1 / 2} \overline{\boldsymbol{U}}^{*}+\boldsymbol{\varepsilon}^{*}\right)^{\prime} \boldsymbol{V}_{U}^{-1}\left(N^{1 / 2} \overline{\boldsymbol{U}}^{*}+\boldsymbol{\varepsilon}^{*}\right),
$$

where

$$
\overline{\boldsymbol{U}}^{*}=\left(\begin{array}{c}
\overline{\boldsymbol{U}}^{(1)}-\overline{\boldsymbol{U}}^{(q)} \\
\vdots \\
\overline{\boldsymbol{U}^{(q-1)}}-\overline{\boldsymbol{U}}^{(q)}
\end{array}\right), \quad \boldsymbol{\varepsilon}^{*}=\left(\begin{array}{c}
\boldsymbol{\varepsilon}^{(1)}-\boldsymbol{\varepsilon}^{(q)} \\
\vdots \\
\boldsymbol{\varepsilon}^{(q-1)}-\boldsymbol{\varepsilon}^{(q)}
\end{array}\right)
$$

and

$$
\begin{aligned}
\boldsymbol{V}_{U}^{-1}= & \operatorname{diag}\left(W_{U}^{(1)}, \ldots, W_{U}^{(q-1)}\right) \\
& -\operatorname{diag}\left(W_{U}^{(1)}, \ldots, W_{U}^{(q-1)}\right)\left\{\left(\mathbf{1}_{q-1} \mathbf{1}_{q-1}^{\prime}\right) \otimes W_{U}^{-1}\right\} \operatorname{diag}\left(W_{U}^{(1)}, \ldots, W_{U}^{(q-1)}\right) .
\end{aligned}
$$

We notice that

$$
\begin{aligned}
\boldsymbol{V}_{U} & =\operatorname{diag}\left\{\left(W_{U}^{(1)}\right)^{-1}, \ldots,\left(W_{U}^{(q-1)}\right)^{-1}\right\}+\left\{\left(\mathbf{1}_{q-1} \mathbf{1}_{q-1}^{\prime}\right) \otimes\left(W_{U}^{(q)}\right)^{-1}\right\} \\
& =\operatorname{diag}\left(\rho_{1}^{-2} S_{U}^{(1)}, \ldots, \rho_{q-1}^{-2} S_{U}^{(q-1)}\right)+\left\{\left(\mathbf{1}_{q-1} \mathbf{1}_{q-1}^{\prime}\right) \otimes \rho_{q}^{-2} S_{U}^{(q)}\right\}
\end{aligned}
$$

by simple matrix algebra, which is an unbiased, consistent estimator of

$$
E\left[N \overline{\boldsymbol{U}}^{*}\left(\overline{\boldsymbol{U}}^{*}\right)^{\prime}\right]=\operatorname{diag}\left(\widetilde{\Sigma}^{(1)}, \ldots, \widetilde{\Sigma}^{(q-1)}\right)+\left\{\left(\mathbf{1}_{q-1} \mathbf{1}_{q-1}^{\prime}\right) \otimes \widetilde{\Sigma}^{(q)}\right\} \equiv \Sigma^{*},
$$

where

$$
\widetilde{\Sigma}^{(a)}=\rho_{a}^{-2} \Sigma^{(a)}
$$

It is easy to see that the limiting nonnul distribution of

$$
T_{J}^{2}=\left(N^{1 / 2} \overline{\boldsymbol{U}}^{*}+\varepsilon^{*}\right)^{\prime} \boldsymbol{V}_{U}^{-1}\left(N^{1 / 2} \overline{\boldsymbol{U}}^{*}+\varepsilon^{*}\right)
$$

is the same as that of

$$
\left(N^{1 / 2} \overline{\boldsymbol{U}}^{*}+\varepsilon^{*}\right)^{\prime}\left(\Sigma^{*}\right)^{-1}\left(N^{1 / 2} \overline{\boldsymbol{U}}^{*}+\varepsilon^{*}\right),
$$


which is asymptotically the noncentral chi-square distribution with $f=p(q-1)$ degrees of freedom and noncentrality parameter $\omega_{\infty}^{2}=\lim _{N \rightarrow \infty}\left(\varepsilon^{*}\right)^{\prime}\left(\Sigma^{*}\right)^{-1} \varepsilon^{*}$ $\left(\lim _{N \rightarrow \infty}\right.$ is the limit when all $N_{a}$ 's are large, in such a way that the total number $N$ of observations goes to infinity) even in a general nonnormal case, including a purely discrete case. This is the standard (first-order) asymptotic theory using the central limit theorem, together with Slutsky's theorem, which is the main reason that James's (1954) statistic $T_{J}^{2}$ proposed under normality can be also applicable for the general distribution of $\boldsymbol{U}^{(a)}$ 's.

In what follows, we always assume, for simplicity, that $\left(\rho_{1}, \ldots, \rho_{q}\right)^{\prime}$ is a fixed $q$-dimensional vector in $\boldsymbol{R}_{+}^{q}$ with $\boldsymbol{R}_{+}=(0, \infty)$, where $\rho_{a}^{2}$ 's are positive rational numbers satisfying $\rho_{1}^{2}+\cdots+\rho_{q}^{2}=1$. In that case, the noncentrality parameter $\omega_{\infty}^{2}$ is equal to $\left(\varepsilon^{*}\right)^{\prime}\left(\Sigma^{*}\right)^{-1} \varepsilon^{*}=\omega^{2}$ (say).

Remark 2. It is convenient for us to introduce a $p q \times 1$ vector and a $p q \times p q$ symmetric matrix

$$
\widetilde{\boldsymbol{\varepsilon}}=\left(\begin{array}{c}
\widetilde{\boldsymbol{\varepsilon}}^{[1]} \\
\vdots \\
\widetilde{\boldsymbol{\varepsilon}}^{[q]}
\end{array}\right), \quad \widetilde{\Lambda}=\left(\begin{array}{cccc}
\widetilde{\Lambda}^{(1,1)} & \widetilde{\Lambda}^{(1,2)} & \ldots & \widetilde{\Lambda}^{(1, q)} \\
\widetilde{\Lambda}^{(2,1)} & \widetilde{\Lambda}^{(2,2)} & \ldots & \widetilde{\Lambda}^{(2, q)} \\
\vdots & \vdots & & \vdots \\
\widetilde{\Lambda}^{(q, 1)} & \widetilde{\Lambda}^{(q, 2)} & \ldots & \widetilde{\Lambda}^{(q, q)}
\end{array}\right),
$$

where

$$
\begin{gathered}
\widetilde{\varepsilon}^{\left[a_{1}\right]}=\left(\widetilde{\varepsilon}_{j_{1}}^{\left[a_{1}\right]}\right)=\Lambda^{\left(a_{1}\right)}\left(\varepsilon^{\left(a_{1}\right)}-\widehat{\bar{\varepsilon}}\right), \\
\widetilde{\Lambda}^{\left(a_{1}, a_{2}\right)}=\left(\widetilde{\Lambda}_{j_{1} j_{2}}^{\left(a_{1}, a_{2}\right)}\right)=\delta_{a_{1} a_{2}} \Lambda^{\left(a_{1}\right)}-\Lambda^{\left(a_{1}\right)} \Lambda^{-1} \Lambda^{\left(a_{2}\right)} \\
\left(a_{1}, a_{2}=1, \ldots, q ; j_{1}, j_{2}=1, \ldots, p\right), \text { with } \\
\widehat{\bar{\varepsilon}}=\Lambda^{-1} \sum_{a=1}^{q} \Lambda^{(a)} \varepsilon^{(a)} .
\end{gathered}
$$

By simple matrix algebra, we obtain

$$
\begin{aligned}
\left(\Sigma^{*}\right)^{-1}= & \operatorname{diag}\left(\Lambda^{(1)}, \ldots, \Lambda^{(q-1)}\right) \\
& -\operatorname{diag}\left(\Lambda^{(1)}, \ldots, \Lambda^{(q-1)}\right)\left\{\left(\mathbf{1}_{q-1} \mathbf{1}_{q-1}^{\prime}\right) \otimes \Lambda^{-1}\right\} \\
& \times \operatorname{diag}\left(\Lambda^{(1)}, \ldots, \Lambda^{(q-1)}\right) \\
= & \left(\begin{array}{cccc}
\widetilde{\Lambda}^{(1,1)} & \widetilde{\Lambda}^{(1,2)} & \ldots & \widetilde{\Lambda}^{(1, q-1)} \\
\widetilde{\Lambda}^{(2,1)} & \widetilde{\Lambda}^{(2,2)} & \ldots & \widetilde{\Lambda}^{(2, q-1)} \\
\vdots & \vdots & & \vdots \\
\widetilde{\Lambda}^{(q-1,1)} & \widetilde{\Lambda}^{(q-1,2)} & \ldots & \widetilde{\Lambda}^{(q-1, q-1)}
\end{array}\right) .
\end{aligned}
$$

Then,

$$
\left(\Sigma^{*}\right)^{-1} \varepsilon^{*}=\left(\begin{array}{c}
\Lambda^{(1)}\left(\varepsilon^{(1)}-\widehat{\bar{\varepsilon}}\right) \\
\vdots \\
\Lambda^{(q-1)}\left(\varepsilon^{(q-1)}-\widehat{\bar{\varepsilon}}\right)
\end{array}\right)=\left(\begin{array}{c}
\widetilde{\boldsymbol{\varepsilon}}^{[1]} \\
\vdots \\
\widetilde{\boldsymbol{\varepsilon}}^{[q-1]}
\end{array}\right)
$$


and

$$
-\sum_{b=1}^{q-1} \widetilde{\varepsilon}^{[b]}=\Lambda^{(q)}\left(\varepsilon^{(q)}-\widehat{\bar{\varepsilon}}\right)=\widetilde{\varepsilon}^{[q]} .
$$

It follows that the noncentrality parameter $\omega^{2}=\left(\varepsilon^{*}\right)^{\prime}\left(\Sigma^{*}\right)^{-1} \varepsilon^{*}$ is expressed as

$$
\begin{aligned}
\omega^{2} & =\left\{\left(\Sigma^{*}\right)^{-1} \varepsilon^{*}\right\}^{\prime} \Sigma^{*}\left\{\left(\Sigma^{*}\right)^{-1} \varepsilon^{*}\right\} \\
& =\sum_{b=1}^{q-1}\left(\widetilde{\varepsilon}^{[b]}\right)^{\prime} \widetilde{\Sigma}^{(b)} \widetilde{\varepsilon}^{[b]}+\sum_{b_{1} b_{2}=1}^{q-1}\left(\widetilde{\varepsilon}^{\left[b_{1}\right]}\right)^{\prime} \widetilde{\Sigma}^{(q)} \widetilde{\varepsilon}^{\left[b_{2}\right]} \\
& =\sum_{a=1}^{q}\left(\varepsilon^{(a)}-\widehat{\bar{\varepsilon}}\right)^{\prime} \Lambda^{(a)}\left(\varepsilon^{(a)}-\widehat{\bar{\varepsilon}}\right) .
\end{aligned}
$$

Furthermore, we notice the relations

$$
-\sum_{b=1}^{q-1} \widetilde{\Lambda}^{(\beta, b)}=\widetilde{\Lambda}^{(\beta, q)}, \quad-\sum_{b=1}^{q-1} \widetilde{\Lambda}^{(b, \beta)}=\widetilde{\Lambda}^{(q, \beta)} \quad(\beta=1, \ldots, q-1)
$$

and

$$
\sum_{b_{1} b_{2}=1}^{q-1} \widetilde{\Lambda}^{\left(b_{1}, b_{2}\right)}=\widetilde{\Lambda}^{(q, q)}
$$

\subsection{Asymptotic expansion}

For any symmetric matrix $A$ of order $p, \operatorname{vech}(A)$ is the $p(p+1) / 2$-dimensional vector formed by stacking the columns of $A$ after deleting the upper triangular part of $A$. The class of population distributions of $\boldsymbol{U}^{(a)}$ 's is restricted to the distributions such that $\mathcal{U}^{(a)}=\left(\boldsymbol{U}^{(a)^{\prime}},\left\{\operatorname{vech}\left(\boldsymbol{U}^{(a)} \boldsymbol{U}^{(a)^{\prime}}-\Sigma^{(a)}\right)\right\}^{\prime}\right)^{\prime}$ satisfies Cramér's condition (e.g. Bhattacharya and Rao (1976, page 207), hereafter abbreviated as $\mathrm{BR})$

$$
\limsup _{\|\xi\| \rightarrow \infty}\left|E\left[\exp \left(\mathrm{i} \boldsymbol{\xi}^{\prime} \mathcal{U}^{(a)}\right)\right]\right|<1 \quad\left(\boldsymbol{\xi} \in \boldsymbol{R}^{p+p(p+1) / 2}\right)
$$

with a finite 8 th absolute moment $E\left[\left\|\boldsymbol{U}^{(a)}\right\|^{8}\right]<\infty$. This is the validity condition for an asymptotic expansion up to $N_{a}^{-1}$ of a smooth function of the sample mean $N_{a}^{-1} \sum_{i=1}^{N_{a}} \mathcal{U}^{(a)}$ (e.g. Bhattacharya and Ghosh (1978) and Chandra and Ghosh (1980)) via fundamental theory due to BR (1976, Theorem 20.1).

We define

$$
\begin{aligned}
& \operatorname{tr}_{a}=\operatorname{tr}\left(I_{p}-\Lambda^{-1} \Lambda^{(a)}\right), \quad \operatorname{tr}_{a a}=\operatorname{tr}\left\{\left(I_{p}-\Lambda^{-1} \Lambda^{(a)}\right)^{2}\right\} \\
& \mathcal{E}_{a}^{(2)}=\left(\varepsilon^{(a)}-\widehat{\bar{\varepsilon}}\right)^{\prime} \Lambda^{(a)}\left(\varepsilon^{(a)}-\widehat{\bar{\varepsilon}}\right) \quad\left(\text { note that } \omega^{2}=\sum_{a=1}^{q} \mathcal{E}_{a}^{(2)}\right),
\end{aligned}
$$




$$
\mathcal{E}_{a a}^{(2)}=\left(\varepsilon^{(a)}-\widehat{\bar{\varepsilon}}\right)^{\prime}\left(\Lambda^{(a)}-\Lambda^{(a)} \Lambda^{-1} \Lambda^{(a)}\right)\left(\varepsilon^{(a)}-\widehat{\bar{\varepsilon}}\right)
$$

for $a=1, \ldots, q$. Further, with

$$
\widetilde{\kappa}_{j_{1}, j_{2}, j_{3}}^{(a)}=\frac{\kappa_{j_{1}, j_{2}, j_{3}}^{(a)}}{\rho_{a}^{4}} \quad \text { and } \quad \widetilde{\kappa}_{j_{1}, j_{2}, j_{3}, j_{4}}^{(a)}=\frac{\kappa_{j_{1}, j_{2}, j_{3}, j_{4}}^{(a)}}{\rho_{a}^{6}}
$$

we define

$$
\begin{aligned}
& K_{3}^{[1]}=\sum_{a=1}^{q} \sum_{j_{1} j_{2} j_{3}=1}^{p} \widetilde{\kappa}_{j_{1}, j_{2}, j_{3}}^{(a)} \widetilde{\Lambda}_{j_{1} j_{2}}^{(a, a)} \widetilde{\varepsilon}_{j_{3}}^{[a]} \equiv \sum_{a=1}^{q} \kappa_{3}^{[1]}(a), \\
& K_{3}^{[3]}=\sum_{a=1}^{q} \sum_{j_{1} j_{2} j_{3}=1}^{p} \widetilde{\kappa}_{j_{1}, j_{2}, j_{3}}^{(a)} \widetilde{\varepsilon}_{j_{1}}^{[a]} \widetilde{\varepsilon}_{j_{2}}^{[a]} \widetilde{\varepsilon}_{j_{3}}^{[a]} \equiv \sum_{a=1}^{q} \kappa_{3}^{[3]}(a), \\
& K_{4}=\sum_{a=1}^{q} \sum_{j_{1} j_{2} j_{3} j_{4}=1}^{p} \widetilde{\kappa}_{j_{1}, j_{2}, j_{3}, j_{4}}^{(a)} \widetilde{\Lambda}_{j_{1} j_{2}}^{(a, a)} \widetilde{\Lambda}_{j_{3} j_{4}}^{(a, a)} \equiv \sum_{a=1}^{q} \kappa_{4}(a), \\
& K_{4}^{[2]}=\sum_{a=1}^{q} \sum_{j_{1} j_{2} j_{3} j_{4}=1}^{p} \widetilde{\kappa}_{j_{1}, j_{2}, j_{3}, j_{4}}^{(a)} \widetilde{\Lambda}_{j_{1} j_{2}}^{(a, a)} \widetilde{\varepsilon}_{j_{3}}^{[a]} \widetilde{\varepsilon}_{j_{4}}^{[a]} \equiv \sum_{a=1}^{q} \kappa_{4}^{[2]}(a), \\
& K_{4}^{[4]}=\sum_{a=1}^{q} \sum_{j_{1} j_{2} j_{3} j_{4}=1}^{p} \widetilde{\kappa}_{j_{1}, j_{2}, j_{3}, j_{4}}^{(a)} \widetilde{\varepsilon}_{j_{1}}^{[a]} \widetilde{\varepsilon}_{j_{2}}^{[a]} \widetilde{\varepsilon}_{j_{3}}^{a]} \widetilde{\varepsilon}_{j_{4}}^{[a]} \equiv \sum_{a=1}^{q} \kappa_{4}^{[4]}(a), \\
& K_{33,1}=\sum_{a a^{\prime}=1}^{q} \sum_{j_{1} j_{2} j_{3} k_{4} k_{5} k_{6}=1}^{p} \widetilde{\kappa}_{j_{1}, j_{2}, j_{3}}^{(a)} \widetilde{\kappa}_{k_{4}, k_{5}, k_{6}}^{\left(a^{\prime}\right)} \widetilde{\Lambda}_{j_{1} k_{4}}^{\left(a, a^{\prime}\right)} \widetilde{\Lambda}_{j_{2} k_{5}}^{\left(a, a^{\prime}\right)} \widetilde{\Lambda}_{j_{3} k_{6}}^{\left(a, a^{\prime}\right)} \equiv \sum_{a a^{\prime}=1}^{q} \kappa_{33,1}\left(a, a^{\prime}\right), \\
& K_{33,2}=\sum_{a a^{\prime}=1}^{q} \sum_{j_{1} j_{2} j_{3} k_{4} k_{5} k_{6}=1}^{p} \widetilde{\kappa}_{j_{1}, j_{2}, j_{3}}^{(a)} \widetilde{\kappa}_{k_{4}, k_{5}, k_{6}}^{\left(a^{\prime}\right)} \widetilde{\Lambda}_{j_{1} j_{2}}^{(a, a)} \widetilde{\Lambda}_{j_{3} k_{4}}^{\left(a, a^{\prime}\right)} \widetilde{\Lambda}_{k_{5} k_{6}}^{\left(a^{\prime}, a^{\prime}\right)} \equiv \sum_{a a^{\prime}=1}^{q} \kappa_{33,2}\left(a, a^{\prime}\right), \\
& K_{33,1}^{[2]}=\sum_{a a^{\prime}=1}^{q} \sum_{j_{1} j_{2} j_{3} k_{4} k_{5} k_{6}=1}^{p} \widetilde{\kappa}_{j_{1}, j_{2}, j_{3}}^{(a)} \widetilde{\kappa}_{k_{4}, k_{5}, k_{6}}^{\left(a^{\prime}\right)} \widetilde{\Lambda}_{j_{1} k_{4}}^{\left(a, a^{\prime}\right)} \widetilde{\Lambda}_{j_{2} k_{5}}^{\left(a, a^{\prime}\right)} \widetilde{\varepsilon}_{j_{3}}^{[a]} \widetilde{\varepsilon}_{k_{6}}^{\left[a^{\prime}\right]} \equiv \sum_{a a^{\prime}=1}^{q} \kappa_{33,1}^{[2]}\left(a, a^{\prime}\right), \\
& K_{33,2}^{[2]}=\sum_{a a^{\prime}=1}^{q} \sum_{j_{1} j_{2} j_{3} k_{4} k_{5} k_{6}=1}^{p} \widetilde{\kappa}_{j_{1}, j_{2}, j_{3}}^{(a)} \widetilde{\kappa}_{k_{4}, k_{5}, k_{6}}^{\left(a^{\prime}\right)} \widetilde{\Lambda}_{j_{1} j_{2}}^{(a, a)} \widetilde{\Lambda}_{j_{3} k_{4}}^{\left(a, a^{\prime}\right)} \widetilde{\varepsilon}_{k_{5}}^{\left[a^{\prime}\right]} \widetilde{\varepsilon}_{k_{6}}^{\left[a^{\prime}\right]} \equiv \sum_{a a^{\prime}=1}^{q} \kappa_{33,2}^{[2]}\left(a, a^{\prime}\right), \\
& \left.K_{33}^{[4]}=\sum_{a a^{\prime}=1}^{q} \sum_{j_{1} j_{2} j_{3} k_{4} k_{5} k_{6}=1}^{p} \widetilde{\kappa}_{j_{1}, j_{2}, j_{3}}^{(a)} \widetilde{\kappa}_{k_{4}, k_{5}, k_{6}}^{\left(a^{\prime}\right)} \widetilde{\Lambda}_{j_{1} k_{4}}^{\left(a, a^{\prime}\right)} \widetilde{\varepsilon}_{j_{2}}^{[a]} \widetilde{\varepsilon}_{j_{3}}^{[a]} \widetilde{\varepsilon}_{k_{5}}\left\{a^{\prime}\right] \widetilde{\varepsilon_{k}} \widetilde{a}_{k_{6}} a^{\prime}\right] \equiv \sum_{a a^{\prime}=1}^{q} \kappa_{33}^{[4]}\left(a, a^{\prime}\right) .
\end{aligned}
$$

The following asymptotic expansion is the nonnormal extension of Ito (1969, (4.13)).

THEOREM 1. Under the local alternative (2.1),

$$
\operatorname{Pr}\left(T_{J}^{2} \leq x\right)=G_{f}\left(x ; \omega^{2}\right)+\sum_{r=1}^{2} \frac{1}{N^{r / 2}} \sum_{\ell=0}^{3 r} \pi_{r, \ell} G_{f+2 \ell}\left(x ; \omega^{2}\right)+\mathrm{o}\left(N^{-1}\right),
$$


where $G_{\nu}\left(x ; \omega^{2}\right)$ denotes the distribution function of the noncentral chi-square distribution with $\nu$ degrees of freedom and noncentrality parameter $\omega^{2}$, and each coefficient is given by

$$
\begin{aligned}
& \pi_{1,0}=-\frac{K_{3}^{[3]}}{6}, \quad \pi_{1,1}=K_{3}^{[1]}, \quad \pi_{1,2}=-K_{3}^{[1]}+\frac{K_{3}^{[3]}}{2}, \quad \pi_{1,3}=-\frac{K_{3}^{[3]}}{3}, \\
& \pi_{2,0}=-\frac{1}{4} \sum_{a=1}^{q} \frac{\left(\operatorname{tr}_{a}\right)^{2}}{\rho_{a}^{2}}-\frac{K_{4}}{4}+\frac{K_{33,1}}{6}+\left(\frac{K_{4}^{[4]}}{24}-\frac{K_{33}^{[4]}}{8}\right)+\frac{\left(K_{3}^{[3]}\right)^{2}}{72}, \\
& \pi_{2,1}=-\frac{1}{2} \sum_{a=1}^{q} \frac{\operatorname{tr}_{a a}}{\rho_{a}^{2}}+\frac{K_{4}}{2}-\frac{K_{33,1}}{2}+\left(\frac{1}{2} \sum_{a=1}^{q} \frac{\mathcal{E}_{a a}^{(2)}}{\rho_{a}^{2}}-K_{4}^{[2]}+K_{33,1}^{[2]}+\frac{K_{33,2}^{[2]}}{2}\right) \\
& +\left(\frac{K_{4}^{[4]}}{12}-\frac{K_{33}^{[4]}}{8}-\frac{K_{3}^{[1]} K_{3}^{[3]}}{6}\right) \\
& \pi_{2,2}=\frac{1}{4} \sum_{a=1}^{q} \frac{2 \operatorname{tr}_{a a}+\left(\operatorname{tr}_{a}\right)^{2}}{\rho_{a}^{2}}-\frac{K_{4}}{4}-\frac{K_{33,2}}{2} \\
& +\left\{-\frac{1}{2} \sum_{a=1}^{q} \frac{3 \mathcal{E}_{a a}^{(2)}+\operatorname{tr}_{a} \mathcal{E}_{a}^{(2)}}{\rho_{a}^{2}}+\frac{3 K_{4}^{[2]}}{2}-K_{33,1}^{[2]}+\frac{\left(K_{3}^{[1]}\right)^{2}}{2}\right\} \\
& +\left\{\frac{1}{4} \sum_{a=1}^{q} \frac{\left(\mathcal{E}_{a}^{(2)}\right)^{2}}{\rho_{a}^{2}}-\frac{3 K_{4}^{[4]}}{8}+\frac{5 K_{33}^{[4]}}{8}+\frac{K_{3}^{[1]} K_{3}^{[3]}}{6}\right\}-\frac{\left(K_{3}^{[3]}\right)^{2}}{12}, \\
& \pi_{2,3}=\frac{K_{33,1}}{3}+\frac{K_{33,2}}{2} \\
& +\left\{\frac{1}{2} \sum_{a=1}^{q} \frac{2 \mathcal{E}_{a a}^{(2)}+\operatorname{tr}_{a} \mathcal{E}_{a}^{(2)}}{\rho_{a}^{2}}-\frac{K_{4}^{[2]}}{2}-K_{33,1}^{[2]}-\frac{3 K_{33,2}^{[2]}}{2}-\left(K_{3}^{[1]}\right)^{2}\right\} \\
& +\left\{-\frac{1}{2} \sum_{a=1}^{q} \frac{\left(\mathcal{E}_{a}^{(2)}\right)^{2}}{\rho_{a}^{2}}+\frac{K_{4}^{[4]}}{3}+\frac{K_{33}^{[4]}}{8}+\frac{K_{3}^{[1]} K_{3}^{[3]}}{2}\right\}+\frac{\left(K_{3}^{[3]}\right)^{2}}{18}, \\
& \pi_{2,4}=\left\{K_{33,1}^{[2]}+K_{33,2}^{[2]}+\frac{\left(K_{3}^{[1]}\right)^{2}}{2}\right\} \\
& +\left\{\frac{1}{4} \sum_{a=1}^{q} \frac{\left(\mathcal{E}_{a}^{(2)}\right)^{2}}{\rho_{a}^{2}}-\frac{K_{4}^{[4]}}{12}-K_{33}^{[4]}-\frac{5 K_{3}^{[1]} K_{3}^{[3]}}{6}\right\}+\frac{\left(K_{3}^{[3]}\right)^{2}}{8}, \\
& \pi_{2,5}=\left(\frac{K_{33}^{[4]}}{2}+\frac{K_{3}^{[1]} K_{3}^{[3]}}{3}\right)-\frac{\left(K_{3}^{[3]}\right)^{2}}{6}, \\
& \pi_{2,6}=\frac{\left(K_{3}^{[3]}\right)^{2}}{18} \text {. }
\end{aligned}
$$

Furthermore, for any $c_{1}, c_{2}, c_{3} \in \boldsymbol{R}$, the adjusted statistics

$$
T_{J, *}^{2}=T_{J}^{2}\left\{1-\frac{2}{N} \sum_{j=1}^{3} c_{j}\left(T_{J}^{2}\right)^{j-1}\right\} \equiv B_{c}\left(T_{J}^{2}\right)
$$


(see Cordeiro and Ferrari (1991)) and

$$
T_{J, * *}^{2}=T_{J}^{2}\left\{1-\frac{2}{N} \sum_{j=1}^{3} c_{j}\left(T_{J}^{2}\right)^{j-1}+\frac{1}{N^{2}} \sum_{j_{1} j_{2}=1}^{3} \frac{j_{1} j_{2} c_{j_{1}} c_{j_{2}}}{j_{1}+j_{2}-1}\left(T_{J}^{2}\right)^{j_{1}+j_{2}-2}\right\}
$$

the latter being a monotone transformation $M B_{c}\left(T_{J}^{2}\right)$ (say) of $T_{J}^{2}$ (see Kakizawa (1996)), admit an asymptotic expansion of the nonnull distribution, as follows:

$$
\begin{aligned}
G_{f}\left(x ; \omega^{2}\right) & +\frac{1}{N^{1 / 2}} \sum_{\ell=0}^{3} \pi_{1, \ell} G_{f+2 \ell}\left(x ; \omega^{2}\right) \\
& +\frac{1}{N} \sum_{\ell=0}^{6}\left(\pi_{2, \ell}+\pi_{\ell}^{c}\right) G_{f+2 \ell}\left(x ; \omega^{2}\right)+\mathrm{o}\left(N^{-1}\right),
\end{aligned}
$$

where

$$
\begin{aligned}
& \pi_{0}^{c}=f c_{1}, \\
& \pi_{1}^{c}=-f c_{1}+f(f+2) c_{2}+c_{1} \omega^{2}, \\
& \pi_{2}^{c}=-f(f+2) c_{2}+f(f+2)(f+4) c_{3}+\left\{-c_{1}+2(f+2) c_{2}\right\} \omega^{2}, \\
& \pi_{3}^{c}=-f(f+2)(f+4) c_{3}+\left\{-2(f+2) c_{2}+3(f+2)(f+4) c_{3}\right\} \omega^{2}+c_{2} \omega^{4}, \\
& \pi_{4}^{c}=-3(f+2)(f+4) c_{3} \omega^{2}+\left\{-c_{2}+3(f+4) c_{3}\right\} \omega^{4}, \\
& \pi_{5}^{c}=-3(f+4) c_{3} \omega^{4}+c_{3} \omega^{6}, \\
& \pi_{6}^{c}=-c_{3} \omega^{6} .
\end{aligned}
$$

Remark 3. If $q=2$, then Theorem 1 is nothing but the multivariate Behrens-Fisher problem that Kakizawa and Iwashita (2005, subsection 5.2) considered.

Remark 4. In the special case $p=1$ with variance $\sigma_{a}^{2}$ and the $s$-th cumulant $\kappa_{s \text { times }}^{(a) \ldots, 1}=\left(\sigma_{a}\right)^{s} \kappa_{s}(s=3,4, \ldots)$, we have

$$
\begin{aligned}
& K_{4}=\kappa_{4} \sum_{a=1}^{q} \rho_{a}^{2}\left(\frac{1}{\rho_{a}^{2}}-\frac{\lambda^{-1}}{\sigma_{a}^{2}}\right)^{2} \equiv \kappa_{4} d_{1}, \\
& K_{33,1}=\kappa_{3}^{2}\left[\sum_{a=1}^{q} \rho_{a}^{2}\left(\frac{1}{\rho_{a}^{2}}-\frac{\lambda^{-1}}{\sigma_{a}^{2}}\right)^{2}-\lambda^{-2} \sum_{a a^{\prime}=1}^{q} \frac{\rho_{a}^{2} \rho_{a^{\prime}}^{2}}{\sigma_{a}^{2} \sigma_{a^{\prime}}^{2}}\left(\frac{1}{\rho_{a}^{2}}-\frac{2 \lambda^{-1}}{\sigma_{a}^{2}}+\frac{\lambda^{-1}}{\sigma_{a} \sigma_{a^{\prime}}}\right)\right] \\
& \equiv \kappa_{3}^{2}\left(d_{1}-d_{3}\right), \\
& K_{33,2}=\kappa_{3}^{2}\left[\sum_{a=1}^{q} \rho_{a}^{2}\left(\frac{1}{\rho_{a}^{2}}-\frac{\lambda^{-1}}{\sigma_{a}^{2}}\right)^{2}-\left\{\sum_{a=1}^{q} \frac{\rho_{a}^{2}}{\sigma_{a}}\left(\frac{1}{\rho_{a}^{2}}-\frac{\lambda^{-1}}{\sigma_{a}^{2}}\right)\right\}^{2}\right] \equiv \kappa_{3}^{2}\left(d_{1}-d_{2}\right),
\end{aligned}
$$


where $\lambda=\sum_{a=1}^{q} \rho_{a}^{2} / \sigma_{a}^{2}$. In that case, an asymptotic expansion (2.6) for the null distribution of James's (1954) statistic coincides with Yanagihara (2000).

Let us rearrange the coefficients of $\pi_{r, \ell}$ 's given by Theorem 1 as follows:

$$
\begin{aligned}
& \pi_{1,0}=\pi_{1,0}^{[3]}, \quad \pi_{1,1}=\pi_{1,1}^{[1]}, \quad \pi_{1,2}=\pi_{1,2}^{[1]}+\pi_{1,2}^{[3]}, \quad \pi_{1,3}=\pi_{1,3}^{[3]}, \\
& \pi_{2,0}=\pi_{2,0}^{[0]}+\pi_{2,0}^{[4]}+\pi_{2,0}^{[6]}, \quad \pi_{2,1}=\pi_{2,1}^{[0]}+\pi_{2,1}^{[2]}+\pi_{2,1}^{[4]}, \\
& \pi_{2,2}=\pi_{2,2}^{[0]}+\pi_{2,2}^{[2]}+\pi_{2,2}^{[4]}+\pi_{2,2}^{[6]}, \quad \pi_{2,3}=\pi_{2,3}^{[0]}+\pi_{2,3}^{[2]}+\pi_{2,3}^{[4]}+\pi_{2,3}^{[6]}, \\
& \pi_{2,4}=\pi_{2,4}^{[2]}+\pi_{2,4}^{[4]}+\pi_{2,4}^{[6]}, \quad \pi_{2,5}=\pi_{2,5}^{[4]}+\pi_{2,5}^{[6]}, \quad \pi_{2,6}=\pi_{2,6}^{[6]},
\end{aligned}
$$

where each $\pi_{r, \ell}^{[d]}$ is a homogeneous polynomial of degree $d=0,1,2,3,4,6$ in $\widetilde{\varepsilon}$. Especially,

$$
\begin{aligned}
& \pi_{2,0} \mid \tilde{\varepsilon}=0=-\frac{1}{4} \sum_{a=1}^{q} \frac{\left(\operatorname{tr}_{a}\right)^{2}}{\rho_{a}^{2}}-\frac{K_{4}}{4}+\frac{K_{33,1}}{6} \equiv-f \vartheta_{1}, \\
& \pi_{2,0}+\left.\pi_{2,1}\right|_{\tilde{\varepsilon}=0}=-\frac{1}{4} \sum_{a=1}^{q} \frac{2 \operatorname{tr}_{a a}+\left(\operatorname{tr}_{a}\right)^{2}}{\rho_{a}^{2}}+\frac{K_{4}}{4}-\frac{K_{33,1}}{3} \equiv-f(f+2) \vartheta_{2}, \\
& \pi_{2,0}+\pi_{2,1}+\pi_{2,2} \mid \tilde{\varepsilon}=0=-\frac{K_{33,1}}{3}-\frac{K_{33,2}}{2} \equiv-f(f+2)(f+4) \vartheta_{3} \quad \text { (say). }
\end{aligned}
$$

Let $\boldsymbol{\vartheta}=\left(\vartheta_{1}, \vartheta_{2}, \vartheta_{3}\right)$. As a corollary of Theorem 1 , we have

Corollary 2. $T_{J}^{2}(\boldsymbol{\vartheta})=B_{\vartheta}\left(T_{J}^{2}\right)$ or $M B_{\vartheta}\left(T_{J}^{2}\right)$ admits an asymptotic expansion

$$
\operatorname{Pr}\left[T_{J}^{2}(\boldsymbol{\vartheta}) \leq x\right]=G_{f}\left(x ; \omega^{2}\right)+\sum_{r=1}^{2} \frac{1}{N^{r / 2}} \sum_{\ell=0}^{3 r} \Pi_{r, \ell} G_{f+2 \ell}\left(x ; \omega^{2}\right)+\mathrm{o}\left(N^{-1}\right)
$$

under the local alternative (2.1), where

$$
\begin{aligned}
& \Pi_{1,0}=\pi_{1,0}^{[3]}, \quad \Pi_{1,1}=\pi_{1,1}^{[1]}, \quad \Pi_{1,2}=\pi_{1,2}^{[1]}+\pi_{1,2}^{[3]}, \quad \Pi_{1,3}=\pi_{1,3}^{[3]}, \\
& \Pi_{2,0}=\pi_{2,0}^{[4]}+\pi_{2,0}^{[6]}, \quad \Pi_{2,1}^{[0]}=\pi_{2,1}^{[2]}+\vartheta_{1} \omega^{2}+\pi_{2,1}^{[4]}, \\
& \Pi_{2,2}=\pi_{2,2}^{[2]}+\left\{-\vartheta_{1}+2(f+2) \vartheta_{2}\right\} \omega^{2}+\pi_{2,2}^{[4]}+\pi_{2,2}^{[6]}, \\
& \Pi_{2,3}=\pi_{2,3}^{[2]}+\left\{-2(f+2) \vartheta_{2}+3(f+2)(f+4) \vartheta_{3}\right\} \omega^{2}+\pi_{2,3}^{[4]}+\vartheta_{2} \omega^{4}+\pi_{2,3}^{[6]}, \\
& \Pi_{2,4}=\pi_{2,4}^{[2]}-3(f+2)(f+4) \vartheta_{3} \omega^{2}+\pi_{2,4}^{[4]}+\left\{-\vartheta_{2}+3(f+4) \vartheta_{3}\right\} \omega^{4}+\pi_{2,4}^{[6]}, \\
& \Pi_{2,5}=\pi_{2,5}^{[4]}-3(f+4) \vartheta_{3} \omega^{4}+\pi_{2,5}^{[6]}+\vartheta_{3} \omega^{6}, \quad \Pi_{2,6}=\pi_{2,6}^{[6]}-\vartheta_{3} \omega^{6} .
\end{aligned}
$$

Especially,

$$
P\left[T_{J}^{2}(\boldsymbol{\vartheta}) \leq x \mid H\right]=G_{f}(x)+\mathrm{o}\left(N^{-1}\right),
$$

where $G_{f}(x)$ denotes the distribution function of the central chi-square distribution with $f$ degrees of freedom. 


\subsection{An improved statistic and its power function}

By virtue of Chibisov (1972) (see also Magdalinos (1992)), constructing an appropriate consistent estimator $\widehat{\boldsymbol{\vartheta}}=\left(\widehat{\vartheta}_{1}, \widehat{\vartheta}_{1}, \widehat{\vartheta}_{3}\right)$ of $\boldsymbol{\vartheta}=\left(\vartheta_{1}, \vartheta_{2}, \vartheta_{3}\right)$ implies that

$$
P\left[T_{J}^{2}(\widehat{\boldsymbol{\vartheta}}) \leq x \mid H\right]=G_{f}(x)+\mathrm{o}\left(N^{-1}\right),
$$

and that the test procedure for rejecting $H: \boldsymbol{\mu}^{(1)}=\cdots=\boldsymbol{\mu}^{(q)}$ if $T_{J}^{2}(\widehat{\boldsymbol{\vartheta}})=B_{\widehat{\vartheta}}\left(T_{J}^{2}\right)$ or $M B_{\widehat{\vartheta}}\left(T_{J}^{2}\right)$ exceeds the $\alpha$ percentile $\chi_{f, \alpha}^{2}$ of the central chi-square distribution of $f$ degrees of freedom has the power under the local alternative (2.1)

$\operatorname{Pr}\left[T_{J}^{2}(\widehat{\boldsymbol{\vartheta}})>\chi_{f, \alpha}^{2}\right]=1-G_{f}\left(\chi_{f, \alpha}^{2} ; \omega^{2}\right)-\sum_{r=1}^{2} \frac{1}{N^{r / 2}} \sum_{\ell=0}^{3 r} \Pi_{r, \ell} G_{f+2 \ell}\left(\chi_{f, \alpha}^{2} ; \omega^{2}\right)+\mathrm{o}\left(N^{-1}\right)$.

This power is also obtained by the size corrected test procedure with the rejection region $T_{J}^{2}>\chi_{f, \alpha}^{2}\left\{1+(2 / N) \sum_{j=1}^{3} \widehat{\vartheta}_{j}\left(\chi_{f, \alpha}^{2}\right)^{j-1}\right\}$ on the basis of the Cornish-Fisher expansion, whose validity can be shown as in Hall (1992, Section 3.5).

It remains to construct a consistent estimator of $\boldsymbol{\vartheta}=\left(\vartheta_{1}, \vartheta_{2}, \vartheta_{3}\right)$. Rewrite three summarized cumulants $K_{4}, K_{33,1}$ and $K_{33,2}$ as

$$
\begin{aligned}
& K_{4}=\sum_{a=1}^{q} \sum_{j_{1} j_{2} j_{3} j_{4}=1}^{p} \frac{E\left[U_{j_{1}}^{(a)} U_{j_{2}}^{(a)} U_{j_{3}}^{(a)} U_{j_{4}}^{(a)}\right]}{\rho_{a}^{6}} \widetilde{\Lambda}_{j_{1} j_{2}}^{(a, a)} \widetilde{\Lambda}_{j_{3} j_{4}}^{(a, a)}-\sum_{a=1}^{q} \frac{2 \operatorname{tr}_{a a}+\left(\operatorname{tr}_{a}\right)^{2}}{\rho_{a}^{2}}, \\
& K_{33,1}=\sum_{a a^{\prime}=1}^{q} \sum_{j_{1} j_{2} j_{3} k_{4} k_{5} k_{6}=1}^{p} \frac{E\left[U_{j_{1}}^{(a)} U_{j_{2}}^{(a)} U_{j_{3}}^{(a)}\right] E\left[U_{k_{4}}^{\left(a^{\prime}\right)} U_{k_{5}}^{\left(a^{\prime}\right)} U_{k_{6}}^{\left(a^{\prime}\right)}\right]}{\rho_{a}^{4} \rho_{a^{\prime}}^{4}} \widetilde{\Lambda}_{j_{1} k_{4}}^{\left(a, a^{\prime}\right)} \widetilde{\Lambda}_{j_{2} k_{5}}^{\left(a, a^{\prime}\right)} \widetilde{\Lambda}_{j_{3} k_{6}}^{\left(a, a^{\prime}\right)}, \\
& K_{33,2}=\sum_{a a^{\prime}=1}^{q} \sum_{j_{1} j_{2} j_{3} k_{4} k_{5} k_{6}=1}^{p} \frac{E\left[U_{j_{1}}^{(a)} U_{j_{2}}^{(a)} U_{j_{3}}^{(a)}\right] E\left[U_{k_{4}}^{\left(a^{\prime}\right)} U_{k_{5}}^{\left(a^{\prime}\right)} U_{k_{6}}^{\left(a^{\prime}\right)}\right]}{\rho_{a}^{4} \rho_{a^{\prime}}^{4}} \widetilde{\Lambda}_{j_{1} j_{2}}^{(a, a)} \widetilde{\Lambda}_{j_{3} k_{4}}^{\left(a, a^{\prime}\right)} \widetilde{\Lambda}_{k_{5} k_{6}}^{\left(a^{\prime}, a^{\prime}\right)} .
\end{aligned}
$$

In view of the definition, a set $\left\{\left(\operatorname{tr}_{a a}, \operatorname{tr}_{a}\right) ; a=1, \ldots, q\right\}$ depends only on $\Lambda^{(a)}=$ $\rho_{a}^{2}\left(\Sigma^{(a)}\right)^{-1}(a=1, \ldots, q)$, which are easily estimable by replacing covariance matrices $\Sigma^{(1)}, \ldots, \Sigma^{(q)}$ by their unbiased estimators $S_{Y}^{(1)}, \ldots, S_{Y}^{(q)}$. That is,

$$
\widehat{\operatorname{tr}}_{a}=\operatorname{tr}\left(I_{p}-W_{Y}^{-1} W_{Y}^{(a)}\right) \quad \text { and } \quad \widehat{\operatorname{tr}}_{a a}=\operatorname{tr}\left\{\left(I_{p}-W_{Y}^{-1} W_{Y}^{(a)}\right)^{2}\right\}
$$

are estimators of $\operatorname{tr}_{a}$ and $\operatorname{tr}_{a a}$, respectively. Furthermore, we can construct estimators of $K_{4}+\sum_{a=1}^{q} \rho_{a}^{-2}\left\{2 \operatorname{tr}_{a a}+\left(\operatorname{tr}_{a}\right)^{2}\right\}=\mathcal{M}_{4}$ (say), $K_{33,1}$ and $K_{33,2}$ as follows:

$$
\begin{aligned}
& \widehat{\mathcal{M}}_{4}=\sum_{a=1}^{q} \frac{1}{\rho_{a}^{6}}\left\{\frac{1}{N_{a}} \sum_{i=1}^{N_{a}}\left(M_{i i}^{(a a)}\right)^{2}\right\}, \\
& \widehat{K}_{33,1}=\sum_{a a^{\prime}=1}^{q} \frac{1}{\rho_{a}^{4} \rho_{a^{\prime}}^{4}}\left\{\frac{1}{N_{a} N_{a^{\prime}}} \sum_{i=1}^{N_{a}} \sum_{i^{\prime}=1}^{N_{a^{\prime}}}\left(M_{i i^{\prime}}^{\left(a a^{\prime}\right)}\right)^{3}\right\}, \\
& \widehat{K}_{33,2}=\sum_{a a^{\prime}=1}^{q} \frac{1}{\rho_{a}^{4} \rho_{a^{\prime}}^{4}}\left\{\frac{1}{N_{a} N_{a^{\prime}}} \sum_{i=1}^{N_{a}} \sum_{i^{\prime}=1}^{N_{a^{\prime}}} M_{i i}^{(a a)} M_{i i^{\prime}}^{\left(a a^{\prime}\right)} M_{i^{\prime} i^{\prime}}^{\left(a^{\prime} a^{\prime}\right)}\right\},
\end{aligned}
$$

where

$$
M_{i i^{\prime}}^{\left(a a^{\prime}\right)}=\left(\boldsymbol{Y}_{i}^{(a)}-\overline{\boldsymbol{Y}}^{(a)}\right)^{\prime}\left(\delta_{a a^{\prime}} W_{Y}^{(a)}-W_{Y}^{(a)} W_{Y}^{-1} W_{Y}^{\left(a^{\prime}\right)}\right)\left(\boldsymbol{Y}_{i^{\prime}}^{\left(a^{\prime}\right)}-\overline{\boldsymbol{Y}}^{\left(a^{\prime}\right)}\right) .
$$




\section{Differential operator approach}

As pointed out in Fujikoshi (2002b) for multivariate test statistics on mean vectors, it is crucial to find a convenient device for giving an asymptotic expansion of the characteristic function according to situations under consideration. Unlike Kano (1995) and Fujikoshi (1997, 2002a, 2002b), our approach for obtaining an asymptotic expansion (2.6) or (2.7) is based on the differential operator developed by Kakizawa and Iwashita $(2005,2008)$ and Kakizawa $(2005,2006)$, as follows:

Notation. Let

$$
\gamma^{*}=\left(\gamma_{i_{1}}^{*}\right)=\left(\begin{array}{c}
\gamma^{(1)} \\
\vdots \\
\gamma^{(q-1)}
\end{array}\right)
$$

be an $f \times 1$ vector of variables and

$$
\Gamma^{*}=\left(\gamma_{i_{1} i_{2}}^{*}\right)=\left(\begin{array}{cccc}
\Gamma^{(1,1)} & \Gamma^{(1,2)} & \cdots & \Gamma^{(1, q-1)} \\
\Gamma^{(2,1)} & \Gamma^{(2,2)} & \cdots & \Gamma^{(2, q-1)} \\
\vdots & \vdots & & \vdots \\
\Gamma^{(q-1,1)} & \Gamma^{(q-1,2)} & \cdots & \Gamma^{(q-1, q-1)}
\end{array}\right)
$$

be an $f \times f$ symmetric matrix of variables, where each $\gamma^{\left(b_{1}\right)}=\left(\gamma_{j_{1}}^{\left(b_{1}\right)}\right)$ and $\Gamma^{\left(b_{1}, b_{2}\right)}=\left(\gamma_{j_{1} j_{2}}^{\left(b_{1}, b_{2}\right)}\right)\left(b_{1}, b_{2}=1, \ldots, q-1\right)$ is a $p \times 1$ vector of variables and a $p \times p$ matrix of variables satisfying $\left(\Gamma^{\left(b_{1}, b_{2}\right)}\right)^{\prime}=\Gamma^{\left(b_{2}, b_{1}\right)}$, respectively. We write

$$
\partial_{i_{1}}^{*}=\frac{\partial}{\partial \gamma_{i_{1}}^{*}} \quad \text { and } \quad \partial_{i_{1} i_{2}}^{*}=\frac{1}{2}\left(1+\delta_{i_{1} i_{2}}\right) \frac{\partial}{\partial \gamma_{i_{1} i_{2}}^{*}} \quad\left(i_{1}, i_{2}=1, \ldots, f\right)
$$

with $\delta_{i_{1} i_{2}}$ being the Kronecker delta, that is, $\delta_{i_{1} i_{2}}=1$ iff $i_{1}=i_{2}$, and 0 otherwise. We define an $f \times 1$ vector of differential operators by

$$
\boldsymbol{\partial}^{*}=\left(\partial_{i_{1}}^{*}\right)=\left(\begin{array}{c}
\boldsymbol{\partial}^{(1)} \\
\vdots \\
\boldsymbol{\partial}^{(q-1)}
\end{array}\right) \quad \text { with } \quad \boldsymbol{\partial}^{\left(b_{1}\right)}=\left(\partial_{j_{1}}^{\left(b_{1}\right)}\right)
$$

an $f \times f$ matrix of differential operators by

$$
\begin{aligned}
\partial^{*} & =\left(\partial_{i_{1} i_{2}}^{*}\right) \\
& =\left(\begin{array}{cccc}
\partial^{(1,1)} & \partial^{(1,2)} & \cdots & \partial^{(1, q-1)} \\
\partial^{(2,1)} & \partial^{(2,2)} & \cdots & \partial^{(2, q-1)} \\
\vdots & \vdots & & \vdots \\
\partial^{(q-1,1)} & \partial^{(q-1,2)} & \cdots & \partial^{(q-1, q-1)}
\end{array}\right) \quad \text { with } \quad \partial^{\left(b_{1}, b_{2}\right)}=\left(\partial_{j_{1} j_{2}}^{\left(b_{1}, b_{2}\right)}\right),
\end{aligned}
$$


a $p \times 1$ vector of differential operators by

$$
\partial^{(q)}=\left(\partial_{j_{1}}^{(q)}\right)=-\sum_{b_{1}=1}^{q-1} \partial^{\left(b_{1}\right)}
$$

and a $p \times p$ matrix of differential operators by

$$
\partial^{(q, q)}=\left(\partial_{j_{1} j_{2}}^{(q, q)}\right)=\sum_{b_{1} b_{2}=1}^{q-1} \partial^{\left(b_{1}, b_{2}\right)}
$$

applied to any analytic function of $\gamma^{*}$ and $\Gamma^{*}$. We write $i=\sqrt{-1}$.

LEMMA 3. Let $h\left(\gamma^{*}, \Gamma^{*}\right)$ be an arbitrary multivariate polynomial of finite degree with coefficients in $\boldsymbol{R}$, which may depend on $N$ but are of order $\mathrm{O}(1)$. Then,

$$
E \exp \left\{\mathrm{i} h\left(N^{1 / 2} \overline{\boldsymbol{U}}^{*}, \boldsymbol{V}_{U}\right)\right\}=\left.\Xi \exp \left\{\mathrm{i} h\left(\boldsymbol{\gamma}^{*}, \Gamma^{*}\right)\right\}\right|_{\gamma^{*}=0, \Gamma^{*}=\Sigma^{*}}+\mathrm{o}\left(N^{-1}\right),
$$

provided that $E\left(\left\|\boldsymbol{U}^{(a)}\right\|^{4}\right)<\infty$, where

$$
\Xi=\Xi_{0}\left\{1+\frac{\Xi_{1}}{N^{1 / 2}}+\frac{1}{N}\left(\Xi_{n}+\Xi_{2}+\frac{1}{2} \Xi_{1}^{2}\right)\right\}
$$

with

$\Xi_{0}=\exp \left\{\frac{1}{2}\left(\boldsymbol{\partial}^{*}\right)^{\prime} \Sigma^{*} \boldsymbol{\partial}^{*}\right\}, \quad \Xi_{1}=\sum_{a=1}^{q} \Xi_{1}^{(a)}, \quad \Xi_{n}=\sum_{a=1}^{q} \frac{\Xi_{n}^{(a)}}{\rho_{a}^{2}}, \quad \Xi_{2}=\sum_{a=1}^{q} \Xi_{2}^{(a)}$,

$\Xi_{1}^{(a)}=\sum_{j_{1} j_{2} j_{3}=1}^{p} \widetilde{\kappa}_{j_{1}, j_{2}, j_{3}}^{(a)}\left(\partial_{j_{1} j_{2}}^{(a, a)} \partial_{j_{3}}^{(a)}+\frac{1}{6} \partial_{j_{1}}^{(a)} \partial_{j_{2}}^{(a)} \partial_{j_{3}}^{(a)}\right)$

$\Xi_{n}^{(a)}=\operatorname{tr}\left(\widetilde{\Sigma}^{(a)} \partial^{(a, a)} \widetilde{\Sigma}^{(a)} \partial^{(a, a)}\right)$,

$\Xi_{2}^{(a)}=\frac{1}{2} \sum_{j_{1} j_{2} j_{3} j_{4}=1}^{p} \widetilde{\kappa}_{j_{1}, j_{2}, j_{3}, j_{4}}^{(a)}\left(\partial_{j_{1} j_{2}}^{(a, a)} \partial_{j_{3} j_{4}}^{(a, a)}+\partial_{j_{1} j_{2}}^{(a, a)} \partial_{j_{3}}^{(a)} \partial_{j_{4}}^{(a)}+\frac{1}{12} \partial_{j_{1}}^{(a)} \partial_{j_{2}}^{(a)} \partial_{j_{3}}^{(a)} \partial_{j_{4}}^{(a)}\right)$.

Proof. In line with Kakizawa and Iwashita (2005), we obtain

$$
\begin{aligned}
E \exp \left\{\mathrm{i} h\left(N^{1 / 2} \overline{\boldsymbol{U}}^{*}, \boldsymbol{V}_{U}\right)\right\} & =E \exp \left\{\mathrm{i} h\left(N^{1 / 2} \overline{\boldsymbol{U}}_{\dagger}^{*}, \boldsymbol{V}_{U_{\dagger}}\right)\right\}+\mathrm{o}\left(N^{-1}\right) \\
& =\left.\Theta \exp \left\{\mathrm{i} h\left(\boldsymbol{\gamma}^{*}, \Gamma^{*}\right)\right\}\right|_{\gamma^{*}=0, \Gamma^{*}=\Sigma^{*}}+\mathrm{o}\left(N^{-1}\right),
\end{aligned}
$$

where $\overline{\boldsymbol{U}}_{\dagger}^{*}$ and $\boldsymbol{V}_{U_{\dagger}}$ are, respectively, defined by $\overline{\boldsymbol{U}}^{*}$ and $\boldsymbol{V}_{U}$ with $\boldsymbol{U}_{i}^{(a)}$, s replaced by truncated random vectors

$$
\boldsymbol{U}_{i}^{\dagger(a)}=\left\{\begin{array}{ll}
\boldsymbol{U}_{i}^{(a)}, & \left\|\boldsymbol{U}_{i}^{(a)}\right\| \leq N_{a}^{1 / 2} \\
\mathbf{0}, & \left\|\boldsymbol{U}_{i}^{(a)}\right\|>N_{a}^{1 / 2}
\end{array} \quad\left(a=1, \ldots, q ; i=1,2, \ldots, N_{a}\right),\right.
$$


and

$$
\Theta=E \exp \left[\left(N^{1 / 2} \overline{\boldsymbol{U}}_{\dagger}^{*}\right)^{\prime} \boldsymbol{\partial}^{*}+\operatorname{tr}\left\{\left(\boldsymbol{V}_{U_{\dagger}}-\Sigma^{*}\right) \partial^{*}\right\}\right] .
$$

Now, we have

$$
\begin{aligned}
\left(N^{1 / 2} \overline{\boldsymbol{U}}_{\dagger}^{*}\right)^{\prime} \boldsymbol{\partial}^{*} & =\sum_{b=1}^{q-1}\left(\rho_{b}^{-1} N_{b}^{1 / 2} \overline{\boldsymbol{U}}_{\dagger}^{(b)}-\rho_{q}^{-1} N_{q}^{1 / 2} \overline{\boldsymbol{U}}_{\dagger}^{(q)}\right)^{\prime} \boldsymbol{\partial}^{(b)} \\
& =\sum_{a=1}^{q}\left(N_{a}^{1 / 2} \overline{\boldsymbol{U}}_{\dagger}^{(a)}\right)^{\prime}\left(\rho_{a}^{-1} \boldsymbol{\partial}^{(a)}\right)
\end{aligned}
$$

and

$$
\begin{aligned}
\operatorname{tr}\left\{\left(\boldsymbol{V}_{U_{\dagger}}-\Sigma^{*}\right) \partial^{*}\right\}= & \operatorname{tr}\left[\operatorname{diag}\left(\rho_{1}^{-2} S_{U_{\dagger}}^{(1)}-\widetilde{\Sigma}^{(1)}, \ldots, \rho_{q-1}^{-2} S_{U_{\dagger}}^{(q-1)}-\widetilde{\Sigma}^{(q-1)}\right) \partial^{*}\right] \\
& +\operatorname{tr}\left[\left\{\left(\mathbf{1}_{q-1} \mathbf{1}_{q-1}^{\prime}\right) \otimes\left(\rho_{q}^{-2} S_{U_{\dagger}}^{(q)}-\widetilde{\Sigma}^{(q)}\right)\right\} \partial^{*}\right] \\
= & \sum_{a=1}^{q} \operatorname{tr}\left\{\left(S_{U_{\dagger}}^{(a)}-\Sigma^{(a)}\right)\left(\rho_{a}^{-2} \partial^{(a, a)}\right)\right\} .
\end{aligned}
$$

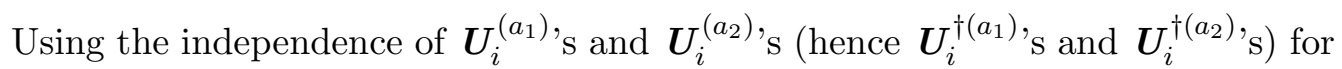
$a_{1} \neq a_{2}$, we obtain

$$
\begin{aligned}
\Theta & =\prod_{a=1}^{q} E \exp \left[\left(N_{a}^{1 / 2} \overline{\boldsymbol{U}}_{\dagger}^{(a)}\right)^{\prime}\left(\rho_{a}^{-1} \boldsymbol{\partial}^{(a)}\right)+\operatorname{tr}\left\{\left(S_{U_{\dagger}}^{(a)}-\Sigma^{(a)}\right)\left(\rho_{a}^{-2} \partial^{(a, a)}\right)\right\}\right] \\
& =\prod_{a=1}^{q} \Theta^{(a)}\left(\rho_{a}^{-1} \boldsymbol{\partial}^{(a)}, \rho_{a}^{-2} \partial^{(a, a)} ; N_{a}\right)+\mathrm{o}\left(N^{-1}\right),
\end{aligned}
$$

with $\Theta^{(a)}(\boldsymbol{\partial}, \partial ; N)$ being the differential operator

$$
\Theta^{(a)}(\boldsymbol{\partial}, \partial ; N)=\exp \left(\frac{1}{2} \boldsymbol{\partial}^{\prime} \Sigma^{(a)} \boldsymbol{\partial}\right)\left[1+\frac{\Theta_{1}^{(a)}}{N^{1 / 2}}+\frac{1}{N}\left\{\Theta_{n}^{(a)}+\Theta_{2}^{(a)}+\frac{1}{2}\left(\Theta_{1}^{(a)}\right)^{2}\right\}\right]
$$

given by Kakizawa and Iwashita (2005), where

$$
\begin{aligned}
& \Theta_{1}^{(a)}=\sum_{j_{1} j_{2} j_{3}=1}^{p} \kappa_{j_{1}, j_{2}, j_{3}}^{(a)}\left(\partial_{j_{1} j_{2}} \partial_{j_{3}}+\frac{1}{6} \partial_{j_{1}} \partial_{j_{2}} \partial_{j_{3}}\right), \quad \Theta_{n}^{(a)}=\operatorname{tr}\left(\Sigma^{(a)} \partial \Sigma^{(a)} \partial\right), \\
& \Theta_{2}^{(a)}=\frac{1}{2} \sum_{j_{1} j_{2} j_{3} j_{4}=1}^{p} \kappa_{j_{1}, j_{2}, j_{3}, j_{4}}^{(a)}\left(\partial_{j_{1} j_{2}} \partial_{j_{3} j_{4}}+\partial_{j_{1} j_{2}} \partial_{j_{3}} \partial_{j_{4}}+\frac{1}{12} \partial_{j_{1}} \partial_{j_{2}} \partial_{j_{3}} \partial_{j_{4}}\right) .
\end{aligned}
$$

Let $i(a)=i+(a-1) p$ for $a=1, \ldots, q$ and $i=1, \ldots, p$. In view of $(3.1)$ and (3.2), we notice

$$
\partial_{i}^{\left(b_{1}\right)}=\partial_{i\left(b_{1}\right)}^{*} \quad \text { and } \quad \partial_{i j}^{\left(b_{1}, b_{2}\right)}=\partial_{i\left(b_{1}\right) j\left(b_{2}\right)}^{*} \quad\left(b_{1}, b_{2}=1, \ldots, q-1\right) .
$$


Following Kakizawa and Iwashita (2005), we consider a multivariate polynomial (the details will be explained in Section 4 below)

$$
H_{0}\left(\gamma^{*}, \Gamma^{*}\right)=\left(\gamma^{*}\right)^{\prime}\left(\Sigma^{*}\right)^{-1} \gamma^{*}+\sum_{\nu=1}^{4} \pi_{\nu}\left(\gamma^{*}\right)^{\prime}\left(\Sigma^{*}\right)^{-1}\left\{\Delta\left(\Gamma^{*}\right)\left(\Sigma^{*}\right)^{-1}\right\}^{\nu} \gamma^{*}
$$

with $\left(\pi_{1}, \pi_{2}, \pi_{3}, \pi_{4}\right)=(-1,1,-3 / 8,9 / 64)$ and $\Delta\left(\Gamma^{*}\right)=\Gamma^{*}-\Sigma^{*}$. It is not difficult to verify the relations

$$
\begin{aligned}
\left.\partial_{j_{1}\left(b_{1}\right) j_{2}\left(b_{2}\right)}^{*} \exp \left\{\mathrm{it} H_{0}\left(\gamma^{*}+\varepsilon^{*}, \Gamma^{*}\right)\right\}\right|_{\Gamma^{*}=\Sigma^{*}} \\
=\left\{\begin{array}{l}
\frac{1}{2}\left(\left[\Sigma^{*}\right]^{j_{1}\left(b_{1}\right) j_{2}\left(b_{2}\right)}-\frac{\left.\partial_{j_{1}\left(b_{1}\right)}^{*} \partial_{j_{2}\left(b_{2}\right)}^{*}\right)}{2 \mathrm{i} t}\right) \\
\quad \times \exp \left\{\mathrm{it}\left(\boldsymbol{\gamma}^{*}+\varepsilon^{*}\right)^{\prime}\left(\Sigma^{*}\right)^{-1}\left(\boldsymbol{\gamma}^{*}+\varepsilon^{*}\right)\right\}, \quad t \neq 0 \\
0, \quad t=0
\end{array}\right.
\end{aligned}
$$

and

$$
\begin{aligned}
& \left.\partial_{j_{1}\left(b_{1}\right) j_{2}\left(b_{2}\right)}^{*} \partial_{j_{3}\left(b_{3}\right) j_{4}\left(b_{4}\right)}^{*} \exp \left\{\mathrm{it} H_{0}\left(\gamma^{*}+\varepsilon^{*}, \Gamma^{*}\right)\right\}\right|_{\Gamma^{*}=\Sigma^{*}} \\
& \left\{\begin{aligned}
\frac{1}{4}[- & \left(\left[\Sigma^{*}\right]^{j_{1}\left(b_{1}\right) j_{3}\left(b_{3}\right)}\left[\Sigma^{*}\right]^{j_{2}\left(b_{2}\right) j_{4}\left(b_{4}\right)}\right. \\
& \left.+\left[\Sigma^{*}\right]^{j_{1}\left(b_{1}\right) j_{4}\left(b_{4}\right)}\left[\Sigma^{*}\right]^{j_{2}\left(b_{2}\right) j_{3}\left(b_{3}\right)}\right) \\
& +\left(\left[\Sigma^{*}\right]^{j_{1}\left(b_{1}\right) j_{2}\left(b_{2}\right)}-\frac{\left.\partial_{j_{1}\left(b_{1}\right)}^{*} \partial_{j_{2}\left(b_{2}\right)}^{*}\right)}{2 \mathrm{i} t}\right) \\
& \left.\times\left(\left[\Sigma^{*}\right]^{j_{3}\left(b_{3}\right) j_{4}\left(b_{4}\right)}-\frac{\left.\partial_{j_{3}\left(b_{3}\right)}^{*} \partial_{j_{4}\left(b_{4}\right)}^{*}\right)}{2 \mathrm{i} t}\right)\right] \\
& \times \exp \left\{\mathrm{i} t\left(\gamma^{*}+\varepsilon^{*}\right)^{\prime}\left(\Sigma^{*}\right)^{-1}\left(\gamma^{*}+\varepsilon^{*}\right)\right\}, \quad t \neq 0 \\
& t=0,
\end{aligned}\right.
\end{aligned}
$$

where

$$
\left[\Sigma^{*}\right]^{j_{1}\left(b_{1}\right) j_{2}\left(b_{2}\right)}=\widetilde{\Lambda}_{j_{1} j_{2}}^{\left(b_{1}, b_{2}\right)}
$$

(see $(2.3))$. We can also apply formulae (see Kakizawa and Iwashita (2005))

$$
\left.\exp \left\{\frac{1}{2}\left(\boldsymbol{\partial}^{*}\right)^{\prime} \Sigma^{*} \boldsymbol{\partial}^{*}\right\} \exp \left\{\mathrm{i} t\left(\boldsymbol{\gamma}^{*}+\boldsymbol{\varepsilon}^{*}\right)^{\prime}\left(\Sigma^{*}\right)^{-1}\left(\boldsymbol{\gamma}^{*}+\boldsymbol{\varepsilon}^{*}\right)\right\}\right|_{\gamma^{*}=0}=\operatorname{ch}_{f}\left(t ; \omega^{2}\right)
$$

and

$$
\begin{aligned}
& \exp \left\{\frac{1}{2}\left(\boldsymbol{\partial}^{*}\right)^{\prime} \Sigma^{*} \boldsymbol{\partial}^{*}\right\} \partial_{j_{1}\left(b_{1}\right)}^{*} \cdots \partial_{j_{v}\left(b_{v}\right)}^{*} \\
& \quad \times\left.\exp \left\{\mathrm{it}\left(\boldsymbol{\gamma}^{*}+\boldsymbol{\varepsilon}^{*}\right)^{\prime}\left(\Sigma^{*}\right)^{-1}\left(\boldsymbol{\gamma}^{*}+\boldsymbol{\varepsilon}^{*}\right)\right\}\right|_{\gamma^{*}=0}
\end{aligned}
$$




$$
=\operatorname{ch}_{f}\left(t ; \omega^{2}\right) Q_{j_{1}\left(b_{1}\right) \ldots j_{v}\left(b_{v}\right)}^{*}\left(\varepsilon^{*} ; \frac{\mathrm{i} t}{1-2 \mathrm{i} t}\right)
$$

$\left(v \in \boldsymbol{N}\right.$ and $\left.b_{1}, \ldots, b_{v} \in\{1, \ldots, q-1\}\right)$, where

$$
\operatorname{ch}_{f}\left(t ; \omega^{2}\right)=(1-2 \mathrm{i} t)^{-f / 2} \exp \left(\frac{\mathrm{i} t \omega^{2}}{1-2 \mathrm{i} t}\right) .
$$

With $\varphi=(1-2 \mathrm{i} t)^{-1}$, we have the general expression of

$$
\begin{aligned}
& Q_{J_{1} \ldots J_{v}}^{*}\left(\varepsilon^{*} ; \frac{\mathrm{i} t}{1-2 \mathrm{i} t}\right) \\
& =\sum_{h=0}^{m}(\varphi-1)^{v-h}\left\langle\frac{v !}{2^{h} h !(v-2 h) !}\right\rangle_{2^{h} \mid 1^{v-2 h}} \\
& \times\left[\Sigma^{*}\right]^{J_{1} J_{2}} \cdots\left[\Sigma^{*}\right]^{J_{2 h-1} J_{2 h}}\left[\left(\Sigma^{*}\right)^{-1} \varepsilon^{*}\right]_{J_{2 h+1}} \cdots\left[\left(\Sigma^{*}\right)^{-1} \varepsilon^{*}\right]_{J_{v}}
\end{aligned}
$$

for $v=2 m(\neq 0)$ or $2 m+1$ with $m$ being a nonnegative interger, where $\left[\Sigma^{*}\right]^{J_{1} J_{2}}$ and $\left[\left(\Sigma^{*}\right)^{-1} \varepsilon^{*}\right]_{J_{1}}$ denote the $\left(J_{1}, J_{2}\right)$-th element of $\left(\Sigma^{*}\right)^{-1}$ (see $(2.3)$ or $\left.(3.7)\right)$ and the $J_{1}$-th element of $\left(\Sigma^{*}\right)^{-1} \varepsilon^{*}$ (see $\left.(2.4)\right)$, respectively. Here, $\langle n\rangle_{2^{h} \mid 1^{v-2 h}}$ before terms with indices means a sum of $n$ similar terms obtained by index permutation, where $v ! /\left\{2^{h} h !(v-2 h) !\right\}$ is the number of the partitions of $\{1, \ldots, v\}$ into $h$ pairs and $v-2 h$ singletons.

Remark 5. Strictly speaking, formulae (3.5) (3.6) and (3.8) only apply when $b_{1}, \ldots, b_{v} \in\{1, \ldots, q-1\}$, but if we interpret operators (3.3) and (3.4) as

$$
\partial_{j_{1}(q)}^{*}=-\sum_{b_{1}=1}^{q-1} \partial_{j_{1}\left(b_{1}\right)}^{*} \quad \text { and } \quad \partial_{j_{1}(q) j_{2}(q)}^{*}=\sum_{b_{1} b_{2}=1}^{q-1} \partial_{j_{1}\left(b_{1}\right) j_{2}\left(b_{2}\right)}^{*},
$$

respectively, they can be shown to hold universally (see Remark 2) even when some $b_{*}$ is equal to $q$. Especially, we have

$$
\begin{aligned}
& \left.\partial_{j_{1}\left(a_{1}\right) j_{2}\left(a_{2}\right)}^{*} \exp \left\{\mathrm{it} H_{0}\left(\gamma^{*}+\varepsilon^{*}, \Gamma^{*}\right)\right\}\right|_{\Gamma^{*}=\Sigma^{*}} \\
& =\left\{\begin{array}{l}
\frac{1}{2}\left(\widetilde{\Lambda}_{j_{1} j_{2}}^{\left(a_{1}, a_{2}\right)}-\frac{\partial_{j_{1}\left(a_{1}\right)}^{*} \partial_{j_{2}\left(a_{2}\right)}^{*}}{2 \mathrm{i} t}\right) \\
\quad \times \exp \left\{\mathrm{i} t\left(\gamma^{*}+\varepsilon^{*}\right)^{\prime}\left(\Sigma^{*}\right)^{-1}\left(\gamma^{*}+\varepsilon^{*}\right)\right\}, \quad t \neq 0 \\
0, \quad t=0
\end{array}\right. \\
& \left.\partial_{j_{1}\left(a_{1}\right) j_{2}\left(a_{2}\right)}^{*} \partial_{j_{3}\left(a_{3}\right) j_{4}\left(a_{4}\right)}^{*} \exp \left\{\mathrm{i} t H_{0}\left(\gamma^{*}+\varepsilon^{*}, \Gamma^{*}\right)\right\}\right|_{\Gamma^{*}=\Sigma^{*}} \\
& =\left\{\begin{array}{l}
\frac{1}{4}\left[-\left(\widetilde{\Lambda}_{j_{1} j_{3}}^{\left(a_{1}, a_{3}\right)} \widetilde{\Lambda}_{j_{2} j_{4}}^{\left(a_{2}, a_{4}\right)}+\widetilde{\Lambda}_{j_{1} j_{4}}^{\left(a_{1}, a_{4}\right)} \widetilde{\Lambda}_{j_{2} j_{3}}^{\left(a_{2}, a_{3}\right)}\right)\right. \\
\left.+\left(\widetilde{\Lambda}_{j_{1} j_{2}}^{\left(a_{1}, a_{2}\right)}-\frac{\left.\partial_{j_{1}\left(a_{1}\right)}^{*} \partial_{j_{2}\left(a_{2}\right)}^{*}\right)}{2 \mathrm{i} t}\right)\left(\widetilde{\Lambda}_{j_{3} j_{4}}^{\left(a_{3}, a_{4}\right)}-\frac{\left.\partial_{j_{3}\left(a_{3}\right)}^{*} \partial_{j_{4}\left(a_{4}\right)}^{*}\right)}{2 \mathrm{i} t}\right)\right]
\end{array}\right. \\
& \times \exp \left\{\mathrm{i} t\left(\gamma^{*}+\varepsilon^{*}\right)^{\prime}\left(\Sigma^{*}\right)^{-1}\left(\gamma^{*}+\varepsilon^{*}\right)\right\}, \quad t \neq 0 \\
& 0, \quad t=0
\end{aligned}
$$


$\left(a_{1}, a_{2}, a_{3}, a_{4} \in\{1, \ldots, q\}\right)$ and

$$
\begin{aligned}
& \exp \left\{\frac{1}{2}\left(\boldsymbol{\partial}^{*}\right)^{\prime} \Sigma^{*} \boldsymbol{\partial}^{*}\right\} \partial_{j_{1}\left(a_{1}\right)}^{*} \cdots \partial_{j_{v}\left(a_{v}\right)}^{*} \exp \left\{\mathrm{it}\left(\boldsymbol{\gamma}^{*}+\varepsilon^{*}\right)^{\prime}\left(\Sigma^{*}\right)^{-1}\left(\boldsymbol{\gamma}^{*}+\boldsymbol{\varepsilon}^{*}\right)\right\} \mid \gamma^{*}=0 \\
& =\operatorname{ch}_{f}\left(t ; \omega^{2}\right) Q_{j_{1} \ldots j_{v}}^{a_{1} \ldots a_{v}} \\
& \left(v=2 m(\neq 0) \quad \text { or } \quad 2 m+1 \quad \text { and } \quad a_{1}, \ldots, a_{v} \in\{1, \ldots, q\}\right),
\end{aligned}
$$

where

$$
\begin{aligned}
Q_{j_{1} \ldots j_{v}}^{a_{1} \ldots a_{v}}= & \sum_{h=0}^{m}(\varphi-1)^{v-h}\left\langle\frac{v !}{2^{h} h !(v-2 h) !}\right\rangle_{2^{h} \mid 1^{v-2 h}} \\
& \times \widetilde{\Lambda}_{j_{1} j_{2}}^{\left(a_{1}, a_{2}\right)} \cdots \widetilde{\Lambda}_{j_{2 h-1} j_{2 h}}^{\left(a_{2 h-1}, a_{2 h}\right)} \widetilde{\varepsilon}_{j_{2 h+1}}^{\left[a_{2 h+1}\right]} \ldots \widetilde{\varepsilon}_{j_{v}}^{\left[a_{v}\right]}
\end{aligned}
$$

\section{Proof of Theorem 1}

Under the local alternative (2.1), we can write

$$
T_{J}^{2}=\left(N^{1 / 2} \overline{\boldsymbol{U}}^{*}+\varepsilon^{*}\right)^{\prime} \boldsymbol{V}_{U}^{-1}\left(N^{1 / 2} \overline{\boldsymbol{U}}^{*}+\boldsymbol{\varepsilon}^{*}\right)
$$

(see $(2.2))$. Motivated by the identity $\left(I_{p}-\Delta+\Delta^{2}\right)\left(I_{p}+\Delta\right)=I_{p}+\Delta^{3}$, we have $\left(I_{p}+\Delta\right)^{-1} \approx I_{p}-\Delta+\Delta^{2}$, provided that $\operatorname{tr}\left(\Delta^{2}\right)$ is sufficently small (we assume that $\Delta$ is a $p \times p$ symmetric matrix). Further, we know

$$
\left(I_{p}-\frac{1}{2} \Delta+\frac{3}{8} \Delta^{2}\right)^{2}=I_{p}-\Delta+\Delta^{2}-\frac{3}{8} \Delta^{3}+\frac{9}{64} \Delta^{4} .
$$

Thus, we set down

$$
\boldsymbol{S}_{\varepsilon}^{*}=\left(I_{p}-\frac{1}{2} \widetilde{\Delta}+\frac{3}{8} \widetilde{\Delta}^{2}\right)\left(\Sigma^{*}\right)^{-1 / 2}\left(N^{1 / 2} \overline{\boldsymbol{U}}^{*}+\varepsilon^{*}\right),
$$

where $\widetilde{\Delta}=\left(\Sigma^{*}\right)^{-1 / 2}\left(\boldsymbol{V}_{U}-\Sigma^{*}\right)\left(\Sigma^{*}\right)^{-1 / 2}$ and $\left(\Sigma^{*}\right)^{-1 / 2}$ is the inverse matrix of the symmetric square root matrix $\left(\Sigma^{*}\right)^{1 / 2}$ of $\Sigma^{*}$.

Writing $\zeta=N^{3 / 2}\left\{T_{J}^{2}-\left(\boldsymbol{S}_{\varepsilon}^{*}\right)^{\prime}\left(\boldsymbol{S}_{\varepsilon}^{*}\right)\right\}$, it is easy to see that $P\left(|\zeta|>N^{1 / 2} \rho_{N}\right)=$ $\mathrm{o}\left(N^{-1}\right)$ for some sequence $\rho_{N} \rightarrow 0$. Actually, we used the following three claims which are stated without proof:

Claim 1. Using BR (1976, Corollary 17.12) or the Edgeworth expansion up to order $N_{a}^{-1}$ of the normalized sum of a sequence of iid random vectors $N_{a}^{-1 / 2} \sum_{i=1}^{N_{a}} \mathcal{U}_{i}^{(a)}$ (e.g. BR (1976, Theorem 20.1)), we have

$$
P\left[\left\|N_{a}^{-1 / 2} \sum_{i=1}^{N_{a}} \mathcal{U}_{i}^{(a)}\right\|>\left(3 \tau_{a}^{2} \log N_{a}\right)^{1 / 2}\right]=\mathrm{o}\left(N_{a}^{-1}\right),
$$

where

$$
\mathcal{U}_{i}^{(a)}=\left(\boldsymbol{U}_{i}^{(a)^{\prime}},\left\{\operatorname{vech}\left(\boldsymbol{U}_{i}^{(a)} \boldsymbol{U}_{i}^{(a)^{\prime}}-\Sigma^{(a)}\right)\right\}^{\prime}\right)^{\prime} \quad\left(i=1, \ldots, N_{a}\right)
$$


are independently distributed according to $\mathcal{U}^{(a)}$ given in Subsection 2.2, and $\tau_{a}^{2}$ is the largest eigenvalue of $\operatorname{Cov}\left(\mathcal{U}^{(a)}\right)$.

Claim 2. Based on the product of the Edgeworth expansions up to order $N_{a}^{-1}$ of the normalized sum $N_{a}^{1 / 2} \bar{U}^{(a)}(a=1, \ldots, q)$ (this step is a consequence of BR (1976, Theorem 20.1) for the equal sample size case; otherwise it is a modification of BR (1976, Theorem 20.6)), $\left(\Sigma^{*}\right)^{-1 / 2} \overline{\boldsymbol{U}}^{*}$ admits a valid Edgeworth exapansion up to order $N^{-1}$, hence

$$
P\left[\left\|\left(\Sigma^{*}\right)^{-1 / 2}\left(N^{1 / 2} \overline{\boldsymbol{U}}^{*}+\varepsilon^{*}\right)\right\|>(3 \log N)^{1 / 2}\right]=\mathrm{o}\left(N^{-1}\right) .
$$

CLAIM 3. $\left\|N_{a}^{-1 / 2} \sum_{i=1}^{N_{a}} \mathcal{U}_{i}^{(a)}\right\| \leq\left(3 \tau_{a}^{2} \log N_{a}\right)^{1 / 2}(a=1, \ldots, q)$ implies that the spectral norm of $\widetilde{\Delta}$ is bounded by $1 / 2$ (say) for all sufficiently large $N$.

By virtue of Chibisov (1972) and Magdalinos (1992), an asymptotic expansion for the nonnull distribution of $T_{J}^{2}$ is the same as that of $\left(\boldsymbol{S}_{\varepsilon}^{*}\right)^{\prime}\left(\boldsymbol{S}_{\varepsilon}^{*}\right)$ up to order $N^{-1}$ when it exists.

We now show that the distribution of $\left(\boldsymbol{S}_{\varepsilon}^{*}\right)^{\prime}\left(\boldsymbol{S}_{\varepsilon}^{*}\right)$ admits a valid asymptotic expansion. Recall

$$
\left(\boldsymbol{S}_{\varepsilon}^{*}\right)^{\prime}\left(\boldsymbol{S}_{\varepsilon}^{*}\right)=H_{0}\left(N^{1 / 2} \overline{\boldsymbol{U}}^{*}+\varepsilon^{*}, \boldsymbol{V}_{U}\right)=\widetilde{T}_{J}^{2} \quad(\text { say }) .
$$

From Lemma 3, the characteristic function of $\widetilde{T}_{J}^{2}$ is expanded as

$$
\begin{aligned}
E\left[\exp \left\{\mathrm{it}\left(\boldsymbol{S}_{\varepsilon}^{*}\right)^{\prime}\left(\boldsymbol{S}_{\varepsilon}^{*}\right)\right\}\right] & =\left.\Xi \exp \left\{\mathrm{it} H_{0}\left(\boldsymbol{\gamma}^{*}+\varepsilon^{*}, \Gamma^{*}\right)\right\}\right|_{\gamma^{*}=0, \Gamma^{*}=\Sigma^{*}}+\mathrm{o}\left(N^{-1}\right) \\
& =\operatorname{ch}_{f}\left(t ; \omega^{2}\right)\left(1+\sum_{r=1}^{2} \frac{1}{N^{r / 2}} \sum_{\ell=0}^{3 r} \pi_{r, \ell} \varphi^{\ell}\right)+\mathrm{o}\left(N^{-1}\right)
\end{aligned}
$$

Details of the calculation of each coefficient are given in Appendix A. On the other hand, since $\left(\boldsymbol{S}_{\varepsilon}^{*}\right)^{\prime}\left(\boldsymbol{S}_{\varepsilon}^{*}\right)$ is a smooth function of $\left\{N_{a}^{-1} \sum_{i=1}^{N_{a}} \mathcal{U}_{i}^{(a)}, a=\right.$ $1, \ldots, q\}$, the distribution of $\left(\boldsymbol{S}_{\varepsilon}^{*}\right)^{\prime}\left(\boldsymbol{S}_{\varepsilon}^{*}\right)$ admits a valid expansion under Cramér's condition (2.5) and moment condition $E\left(\left\|\mathcal{U}^{(a)}\right\|^{4}\right)<\infty$, hence $E\left(\left\|\boldsymbol{U}^{(a)}\right\|^{8}\right)<\infty$. More precisely, based on the valid Edgeworth expansion up to order $N^{-1}$ of $\left\{N_{a}^{-1 / 2} \sum_{i=1}^{N_{a}} \mathcal{U}_{i}^{(a)}, a=1, \ldots, q\right\}$ (e.g. BR (1976, Theorems 20.1 and 20.6)), together with the B-G transformation argument (e.g. Bhattacharya and Ghosh (1978) and Bhattacharya and Denker (1990, Section 2 in Part I)), we can show that there exist polynomials $q_{r}(\boldsymbol{y})$ in $f=p(q-1)$ variables $y_{1}, \ldots, y_{f}$ (the coefficients in the $q_{r}(\boldsymbol{y})$ 's are independent of $\left.N\right)$, such that

$$
\begin{aligned}
\sup _{|t| \leq K} \mid E\left[\exp \left\{\operatorname{it}\left(\boldsymbol{S}_{\varepsilon}^{*}\right)^{\prime}\left(\boldsymbol{S}_{\varepsilon}^{*}\right)\right\}\right] \\
\quad-\int_{\mathrm{R}^{f}} \exp \left(\mathrm{i} t \boldsymbol{y}^{\prime} \boldsymbol{y}\right) \phi_{I_{f}}\left(\boldsymbol{y}-\left(\Sigma^{*}\right)^{-1 / 2} \varepsilon^{*}\right)\left\{1+\sum_{r=1}^{2} \frac{q_{r}(\boldsymbol{y})}{N^{r / 2}}\right\} d \boldsymbol{y} \mid=\mathrm{o}\left(N^{-1}\right)
\end{aligned}
$$


for any $K>0$ and

$$
\sup _{A \in \mathcal{A}}\left|\operatorname{Pr}\left(\boldsymbol{S}_{\varepsilon}^{*} \in A\right)-\int_{A} \phi_{I_{f}}\left(\boldsymbol{y}-\left(\Sigma^{*}\right)^{-1 / 2} \varepsilon^{*}\right)\left\{1+\sum_{r=1}^{2} \frac{q_{r}(\boldsymbol{y})}{N^{r / 2}}\right\} d \boldsymbol{y}\right|=\mathrm{o}\left(N^{-1}\right)
$$

for every class $\mathcal{A} \subset \mathcal{B}^{f}$ satisfying

$$
\sup _{A \in \mathcal{A}} \int_{(\partial A)^{\eta}} \phi_{I_{f}}\left(\boldsymbol{y}-\left(\Sigma^{*}\right)^{-1 / 2} \varepsilon^{*}\right) d \boldsymbol{y}=\mathrm{O}(\eta)
$$

as $\eta \rightarrow 0$, where $\phi_{I_{f}}(\boldsymbol{y})$ is the probability density function of $N_{f}\left(\mathbf{0}, I_{f}\right)$ and $(\partial A)^{\eta}$ is the set of points within a distance $\eta$ from boundary of $A$, denoted by $\partial A$. Note that the set

$$
A_{x}=\left\{\boldsymbol{y} \in \boldsymbol{R}^{f}: \boldsymbol{y}^{\prime} \boldsymbol{y} \leq x\right\} \quad(x>0)
$$

is convex and by BR (1976, Theorem 3.1) the class $\mathcal{C}$ of all Borel measurable convex subsets of $\boldsymbol{R}^{f}$ satisfies (4.3). In principle, there exist coefficients $\widetilde{\pi}_{r, \ell}$, independent of $N$, such that

$$
\begin{aligned}
\int_{A_{x}} \phi_{I_{f}}\left(\boldsymbol{y}-\left(\Sigma^{*}\right)^{-1 / 2} \varepsilon^{*}\right)\left\{1+\sum_{r=1}^{2} \frac{q_{r}(\boldsymbol{y})}{N^{r / 2}}\right\} d \boldsymbol{y} \\
=G_{f}\left(x ; \omega^{2}\right)+\sum_{r=1}^{2} \frac{1}{N^{r / 2}} \sum_{\ell=0}^{\operatorname{deg}\left(q_{r}\right)} \widetilde{\pi}_{r, \ell} G_{f+2 \ell}\left(x ; \omega^{2}\right)
\end{aligned}
$$

and

$$
\begin{aligned}
\int_{\mathrm{R}^{f}} & \exp \left(\mathrm{i} t \boldsymbol{y}^{\prime} \boldsymbol{y}\right) \phi_{I_{f}}\left(\boldsymbol{y}-\left(\Sigma^{*}\right)^{-1 / 2} \varepsilon^{*}\right)\left\{1+\sum_{r=1}^{2} \frac{q_{r}(\boldsymbol{y})}{N^{r / 2}}\right\} d \boldsymbol{y} \\
= & \int_{\mathrm{R}} \exp (\mathrm{i} t x)\left\{g_{f}\left(x ; \omega^{2}\right)+\sum_{r=1}^{2} \frac{1}{N^{r / 2}} \sum_{\ell=0}^{\operatorname{deg}\left(q_{r}\right)} \widetilde{\pi}_{r, \ell} g_{f+2 \ell}\left(x ; \omega^{2}\right)\right\} d x,
\end{aligned}
$$

where $g_{\nu}\left(x ; \omega^{2}\right)$ is the probability density function of the noncentral chi-square distribution with $\nu$ degrees of freedom and noncentrality parameter $\omega^{2}$. The unicity property of the Fourier-Stieltjes transform then implies that a formal inversion of (4.2) must be valid.

To deal with $T_{J, *}^{2}$ and $T_{J, * *}^{2}$, we consider

$$
\widetilde{T}_{J, *}^{2}=\left(\boldsymbol{S}_{\varepsilon}^{*}\right)^{\prime}\left[1-\frac{2}{N} \sum_{j=1}^{3} c_{j}\left\{\left(\boldsymbol{S}_{\varepsilon}^{*}\right)^{\prime}\left(\boldsymbol{S}_{\varepsilon}^{*}\right)\right\}^{j-1}\right]\left(\boldsymbol{S}_{\varepsilon}^{*}\right)
$$

and

$$
\widetilde{T}_{J, * *}^{2}=\left(\boldsymbol{S}_{\varepsilon}^{*}\right)^{\prime}\left[1-\frac{2}{N} \sum_{j=1}^{3} c_{j}\left\{\left(\boldsymbol{S}_{\varepsilon}^{*}\right)^{\prime}\left(\boldsymbol{S}_{\varepsilon}^{*}\right)\right\}^{j-1}\right.
$$




$$
\left.+\frac{1}{N^{2}} \sum_{j_{1} j_{2}=1}^{3} \frac{j_{1} j_{2} c_{j_{1}} c_{j_{2}}}{j_{1}+j_{2}-1}\left\{\left(\boldsymbol{S}_{\varepsilon}^{*}\right)^{\prime}\left(\boldsymbol{S}_{\varepsilon}^{*}\right)\right\}^{j_{1}+j_{2}-2}\right]\left(\boldsymbol{S}_{\varepsilon}^{*}\right)
$$

for which we can show that $N^{3 / 2}\left(T_{J, *}^{2}-\widetilde{T}_{J, *}^{2}\right)$ and $N^{3 / 2}\left(T_{J, * *}^{2}-\widetilde{T}_{J, * *}^{2}\right)$ have the same property as $\zeta=N^{3 / 2}\left\{T_{J}^{2}-\left(\boldsymbol{S}_{\varepsilon}^{*}\right)^{\prime}\left(\boldsymbol{S}_{\varepsilon}^{*}\right)\right\}$. We next define

$$
\boldsymbol{S}_{c, \varepsilon}^{*}=\left[1-\frac{1}{N} \sum_{j=1}^{3} c_{j}\left\{\left(\boldsymbol{S}_{\varepsilon}^{*}\right)^{\prime}\left(\boldsymbol{S}_{\varepsilon}^{*}\right)\right\}^{j-1}\right]\left(\boldsymbol{S}_{\varepsilon}^{*}\right),
$$

which is also a smooth function of $\left\{N_{a}^{-1} \sum_{i=1}^{N_{a}} \mathcal{U}_{i}^{(a)}, a=1, \ldots, q\right\}$. Write

$$
\zeta_{*}=N^{3 / 2}\left\{\widetilde{T}_{J, *}^{2}-\left(\boldsymbol{S}_{c, \varepsilon}^{*}\right)^{\prime}\left(\boldsymbol{S}_{c, \varepsilon}^{*}\right)\right\} \quad \text { and } \quad \zeta_{* *}=N^{3 / 2}\left\{\widetilde{T}_{J, * *}^{2}-\left(\boldsymbol{S}_{c, \varepsilon}^{*}\right)^{\prime}\left(\boldsymbol{S}_{c, \varepsilon}^{*}\right)\right\} .
$$

Noting $P\left[\left\|\boldsymbol{S}_{\varepsilon}^{*}\right\|>(3 \log N)^{1 / 2}\right]=\mathrm{o}\left(N^{-1}\right)$, it is easy to see that $\zeta=\zeta_{*}$ or $\zeta_{* *}$ satisfies $P\left(|\zeta|>N^{1 / 2} \rho_{N}^{\prime}\right)=\mathrm{o}\left(N^{-1}\right)$ for some sequence $\rho_{N}^{\prime} \rightarrow 0$. By virtue of Chibisov (1972), an asymptotic expansion of the nonnull distribution of $T_{J, *}^{2}$ or $T_{J, * *}^{2}$ is the same as that of $\left(\boldsymbol{S}_{c, \varepsilon}^{*}\right)^{\prime}\left(\boldsymbol{S}_{c, \varepsilon}^{*}\right)$ up to order $N^{-1}$. Using the validity of the Edgeworth expansion of $\boldsymbol{S}_{c, \varepsilon}^{*}$, the existence of an asymptotic expansion of the distribution of $\left(\boldsymbol{S}_{c, \varepsilon}^{*}\right)^{\prime}\left(\boldsymbol{S}_{c, \varepsilon}^{*}\right)$ is guaranteed, as in the proof for $\left(\boldsymbol{S}_{\varepsilon}^{*}\right)^{\prime}\left(\boldsymbol{S}_{\varepsilon}^{*}\right)$. Although the Edgeworth expansion of $\boldsymbol{S}_{c, \varepsilon}^{*}$ may be given explicitly, it is further required to calculate its integral over the convex set $A_{x}$. This is the reason why we consider an indirect way to evaluate the characteristic function of

$$
\left(\boldsymbol{S}_{c, \varepsilon}^{*}\right)^{\prime}\left(\boldsymbol{S}_{c, \varepsilon}^{*}\right)=\widetilde{T}_{J}^{2}\left[1-\frac{2}{N} \sum_{j=1}^{3} c_{j}\left(\widetilde{T}_{J}^{2}\right)^{j-1}+\frac{1}{N^{2}}\left\{\sum_{j=1}^{3} c_{j}\left(\widetilde{T}_{J}^{2}\right)^{j-1}\right\}^{2}\right]
$$

by means of the differential operator given in Lemma 3, and to use the uniqueness theorem of the Fourier-Stieltjes transform.

Let

$$
H\left(\gamma^{*}, \Gamma^{*}\right)=H_{0}\left(\gamma^{*}, \Gamma^{*}\right)-\frac{2}{N} H_{1}\left(\gamma^{*}, \Gamma^{*}\right)+\frac{1}{N^{2}} H_{2}\left(\gamma^{*}, \Gamma^{*}\right),
$$

where

$$
H_{1}\left(\gamma^{*}, \Gamma^{*}\right)=\sum_{j=1}^{3} c_{j}\left\{H_{0}\left(\gamma^{*}, \Gamma^{*}\right)\right\}^{j}
$$

and

$$
H_{2}\left(\gamma^{*}, \Gamma^{*}\right)=\sum_{j k=1}^{3} c_{j} c_{k}\left\{H_{0}\left(\gamma^{*}, \Gamma^{*}\right)\right\}^{j+k-1} .
$$

Lemma 3 enables us to evaluate

(4.4) $E\left[\exp \left\{\operatorname{it}\left(\boldsymbol{S}_{c, \varepsilon}^{*}\right)^{\prime}\left(\boldsymbol{S}_{c, \varepsilon}^{*}\right)\right\}\right]$ 


$$
\begin{aligned}
& =\left.\Xi \exp \left\{\operatorname{it} H\left(\gamma^{*}+\varepsilon^{*}, \Gamma^{*}\right)\right\}\right|_{\gamma^{*}=0, \Gamma^{*}=\Sigma^{*}}+\mathrm{o}\left(N^{-1}\right) \\
& =\operatorname{ch}_{f}\left(t ; \omega^{2}\right)\left\{1+\frac{1}{N^{1 / 2}} \sum_{\ell=0}^{3} \pi_{1, \ell} \varphi^{\ell}+\frac{1}{N} \sum_{\ell=0}^{6}\left(\pi_{2, \ell}+\pi_{\ell}^{c}\right) \varphi^{\ell}\right\}+\mathrm{o}\left(N^{-1}\right) .
\end{aligned}
$$

Details of the calculation of each coefficient are given in Appendix B.

Appendix A: Evaluation of (4.2)

Let $t \neq 0$, hence $\varphi \equiv(1-2 \mathrm{i} t)^{-1} \neq 1$. With help of Remark 5 , we have only to evaluate

$$
\begin{aligned}
& C_{3}(t)=\left.\Xi_{0} \Xi_{1} e\left(\mathrm{i} t H_{0}\right)\right|_{\gamma^{*}=0, \Gamma^{*}=\Sigma^{*}}, \\
& C_{n}(t)=\left.\Xi_{0} \Xi_{n} e\left(\mathrm{i} t H_{0}\right)\right|_{\gamma^{*}=0, \Gamma^{*}=\Sigma^{*}}, \\
& C_{4}(t)=\left.\Xi_{0} \Xi_{2} e\left(\mathrm{i} t H_{0}\right)\right|_{\gamma^{*}=0, \Gamma^{*}=\Sigma^{*}}, \\
& C_{33}(t)=\left.\frac{1}{2} \Xi_{0} \Xi_{1}^{2} e\left(\mathrm{i} t H_{0}\right)\right|_{\gamma^{*}=0, \Gamma^{*}=\Sigma^{*}},
\end{aligned}
$$

where

$$
e\left(\mathrm{i} t H_{0}\right)=\exp \left\{\mathrm{i} t H_{0}\left(\gamma^{*}+\varepsilon^{*}, \Gamma^{*}\right)\right\} .
$$

The final results show that the formulae remain valid even if $t=0$.

We first evaluate $C_{3}(t)=\sum_{a=1}^{q}\left\{C_{3,1}^{(a)}(t)+C_{3,2}^{(a)}(t)\right\}$, where

$$
\begin{aligned}
C_{3,1}^{(a)}(t) & =\left.\Xi_{0} \sum_{j_{1} j_{2} j_{3}=1}^{p} \widetilde{\kappa}_{j_{1}, j_{2}, j_{3}}^{(a)} \partial_{j_{1}(a) j_{2}(a)}^{*} \partial_{j_{3}(a)}^{*} e\left(\mathrm{i} t H_{0}\right)\right|_{\gamma^{*}=0, \Gamma^{*}=\Sigma^{*}} \\
& =\frac{\operatorname{ch}_{f}\left(t ; \omega^{2}\right)}{2} \sum_{j_{1} j_{2} j_{3}=1}^{p} \widetilde{\kappa}_{j_{1}, j_{2}, j_{3}}^{(a)}\left(\widetilde{\Lambda}_{j_{1} j_{2}}^{(a, a)} Q_{j_{3}}^{a}-\frac{\varphi}{\varphi-1} Q_{j_{1} j_{2} j_{3}}^{a}\right) \\
C_{3,2}^{(a)}(t) & =\left.\frac{\Xi_{0}}{6} \sum_{j_{1} j_{2} j_{3}=1}^{p} \widetilde{\kappa}_{j_{1}, j_{2}, j_{3}}^{(a)} \partial_{j_{1}(a)}^{*} \partial_{j_{2}(a)}^{*} \partial_{j_{3}(a)}^{*} e\left(\mathrm{i} t H_{0}\right)\right|_{\gamma^{*}=0, \Gamma^{*}=\Sigma^{*}} \\
& =\frac{\operatorname{ch}_{f}\left(t ; \omega^{2}\right)}{6} \sum_{j_{1}}^{p} \widetilde{\kappa}_{j_{2} j_{3}=1}^{(a)}{ }_{j_{1}, j_{2}, j_{3}} Q_{j_{1} j_{2} j_{3}}^{a} .
\end{aligned}
$$

Using symmetricity of $\kappa_{j_{1}, j_{2}, j_{3}}^{(a)}$ under permutation, we have

$$
\sum_{j_{1} j_{2} j_{3}=1}^{p} \widetilde{\kappa}_{j_{1}, j_{2}, j_{3}}^{(a)} Q_{j_{1} j_{2} j_{3}}^{a \underset{a}{a}}=3(\varphi-1)^{2} \kappa_{3}^{[1]}(a)+(\varphi-1)^{3} \kappa_{3}^{[3]}(a)
$$

Also, we have

$$
\sum_{j_{1} j_{2} j_{3}=1}^{p} \widetilde{\kappa}_{j_{1}, j_{2}, j_{3}}^{(a)} \widetilde{\Lambda}_{j_{1} j_{2}}^{(a, a)} Q_{j_{3}}^{a}=(\varphi-1) \kappa_{3}^{[1]}(a)
$$

We obtain

$$
\sum_{a=1}^{q} C_{3,1}^{(a)}(t)=\frac{1}{2}\left[(\varphi-1) K_{3}^{[1]}-\varphi\left\{3(\varphi-1) K_{3}^{[1]}+(\varphi-1)^{2} K_{3}^{[3]}\right\}\right] \operatorname{ch}_{f}\left(t ; \omega^{2}\right)
$$




$$
\sum_{a=1}^{q} C_{3,2}^{(a)}(t)=\frac{1}{6}\left\{3(\varphi-1)^{2} K_{3}^{[1]}+(\varphi-1)^{3} K_{3}^{[3]}\right\} \operatorname{ch}_{f}\left(t ; \omega^{2}\right),
$$

which has the same form as Hotelling's one-sample $T^{2}$ (see Kakizawa and Iwashita (2005)). By collecting terms of $\varphi^{\ell}$, we immediately obtain

$$
\sum_{a=1}^{q}\left\{C_{3,1}^{(a)}(t)+C_{3,2}^{(a)}(t)\right\}=\sum_{\ell=0}^{3} \pi_{1, \ell} \varphi^{\ell} \operatorname{ch}_{f}\left(t ; \omega^{2}\right) .
$$

We next evaluate $C_{n}(t)=\sum_{a=1}^{q} \rho_{a}^{-2} C_{n}^{(a)}(t)$ and $C_{4}(t)=\sum_{a=1}^{q}\left\{C_{4,1}^{(a)}(t)+\right.$ $\left.C_{4,2}^{(a)}(t)+C_{4,3}^{(a)}(t)\right\}$, where

$$
\begin{aligned}
& C_{n}^{(a)}(t)=\left.\Xi_{0} \sum_{j_{1} j_{2} j_{3} j_{4}=1}^{p} \widetilde{\Sigma}_{j_{2} j_{3}}^{(a)} \widetilde{\Sigma}_{j_{4} j_{1}}^{(a)} \partial_{j_{1}(a) j_{2}(a)}^{*} \partial_{j_{3}(a) j_{4}(a)}^{*} e\left(\mathrm{i} t H_{0}\right)\right|_{\gamma^{*}=0, \Gamma^{*}=\Sigma^{*}} \\
& =\frac{\operatorname{ch}_{f}\left(t ; \omega^{2}\right)}{4} \sum_{j_{1} j_{2} j_{3} j_{4}=1}^{p} \widetilde{\Sigma}_{j_{2} j_{3}}^{(a)} \widetilde{\Sigma}_{j_{4} j_{1}}^{(a)} \\
& \times\left[-\left(\widetilde{\Lambda}_{j_{1} j_{3}}^{(a, a)} \widetilde{\Lambda}_{j_{2} j_{4}}^{(a, a)}+\widetilde{\Lambda}_{j_{1} j_{4}}^{(a, a)} \widetilde{\Lambda}_{j_{2} j_{3}}^{(a, a)}\right)+\widetilde{\Lambda}_{j_{1} j_{2}}^{(a, a)} \widetilde{\Lambda}_{j_{3} j_{4}}^{(a, a)}\right. \\
& \left.-\frac{\varphi}{\varphi-1}\left(\widetilde{\Lambda}_{j_{3} j_{4}}^{(a, a)} Q_{j_{1} j_{2}}^{a a}+\widetilde{\Lambda}_{j_{1} j_{2}}^{(a, a)} Q_{j_{3} j_{4}}^{a a}\right)+\frac{\varphi^{2}}{(\varphi-1)^{2}} Q_{j_{1} j_{2} j_{3} j_{4}}^{a a}\right], \\
& C_{4,1}^{(a)}(t)=\left.\frac{\Xi_{0}}{2} \sum_{j_{1} j_{2} j_{3} j_{4}=1}^{p} \widetilde{\kappa}_{j_{1}, j_{2}, j_{3}, j_{4}}^{(a)} \partial_{j_{1}(a) j_{2}(a)}^{*} \partial_{j_{3}(a) j_{4}(a)}^{*} e\left(\mathrm{i} t H_{0}\right)\right|_{\gamma^{*}=0, \Gamma^{*}=\Sigma^{*}} \\
& =\frac{\operatorname{ch}_{f}\left(t ; \omega^{2}\right)}{8} \sum_{j_{1} j_{2} j_{3} j_{4}=1}^{p} \widetilde{\kappa}_{j_{1}, j_{2}, j_{3}, j_{4}}^{(a)} \\
& \times\left[-\left(\widetilde{\Lambda}_{j_{1} j_{3}}^{(a, a)} \widetilde{\Lambda}_{j_{2} j_{4}}^{(a, a)}+\widetilde{\Lambda}_{j_{1} j_{4}}^{(a, a)} \widetilde{\Lambda}_{j_{2} j_{3}}^{(a, a)}\right)+\widetilde{\Lambda}_{j_{1} j_{2}}^{(a, a)} \widetilde{\Lambda}_{j_{3} j_{4}}^{(a, a)}\right. \\
& \left.-\frac{\varphi}{\varphi-1}\left(\widetilde{\Lambda}_{j_{3} j_{4}}^{(a, a)} Q_{j_{1} j_{2}}^{a a}+\widetilde{\Lambda}_{j_{1} j_{2}}^{(a, a)} Q_{j_{3} j_{4}}^{a a}\right)+\frac{\varphi^{2}}{(\varphi-1)^{2}} Q_{j_{1} j_{2} j_{3} j_{4}}^{a a}\right] \\
& C_{4,2}^{(a)}(t)=\left.\frac{\Xi_{0}}{2} \sum_{j_{1} j_{2} j_{3} j_{4}=1}^{p} \widetilde{\kappa}_{j_{1}, j_{2}, j_{3}, j_{4}}^{(a)} \partial_{j_{1}(a) j_{2}(a)}^{*} \partial_{j_{3}(a)}^{*} \partial_{j_{4}(a)}^{*} e\left(\mathrm{i} t H_{0}\right)\right|_{\gamma^{*}=0, \Gamma^{*}=\Sigma^{*}} \\
& =\frac{\operatorname{ch}_{f}\left(t ; \omega^{2}\right)}{4} \sum_{j_{1} j_{2} j_{3} j_{4}=1}^{p} \widetilde{\kappa}_{j_{1}, j_{2}, j_{3}, j_{4}}^{(a)}\left(\widetilde{\Lambda}_{j_{1} j_{2}}^{(a, a)} Q_{j_{3} j_{4}}^{a a}-\frac{\varphi}{\varphi-1} Q_{j_{1} j_{2} j_{3} j_{4}}^{a \underset{a}{a} a}\right), \\
& C_{4,3}^{(a)}(t)=\left.\frac{\Xi_{0}}{24} \sum_{j_{1} j_{2} j_{3} j_{4}=1}^{p} \widetilde{\kappa}_{j_{1}, j_{2}, j_{3}, j_{4}}^{(a)} \partial_{j_{1}(a)}^{*} \partial_{j_{2}(a)}^{*} \partial_{j_{3}(a)}^{*} \partial_{j_{4}(a)}^{*} e\left(\mathrm{i} t H_{0}\right)\right|_{\gamma^{*}=0, \Gamma^{*}=\Sigma^{*}} \\
& =\frac{\operatorname{ch}_{f}\left(t ; \omega^{2}\right)}{24} \sum_{j_{1} j_{2} j_{3} j_{4}=1}^{p} \widetilde{\kappa}_{j_{1}, j_{2}, j_{3}, j_{4}}^{(a)} Q_{j_{1} j_{2} j_{3} j_{4}}^{a \text { a } a} .
\end{aligned}
$$


It is straightforward to see that

$$
\begin{aligned}
C_{n}^{(a)}(t)= & \frac{\operatorname{ch}_{f}\left(t ; \omega^{2}\right)}{4} \sum_{j_{1} j_{2} j_{3} j_{4}=1}^{p} \widetilde{\Sigma}_{j_{2} j_{3}}^{(a)} \widetilde{\Sigma}_{j_{4} j_{1}}^{(a)} \\
& \times\left[-\widetilde{\Lambda}_{j_{1} j_{4}}^{(a, a)} \widetilde{\Lambda}_{j_{2} j_{3}}^{(a, a)}-2 \varphi \widetilde{\Lambda}_{j_{3} j_{4}}^{(a, a)}\left\{\widetilde{\Lambda}_{j_{1} j_{2}}^{(a, a)}+(\varphi-1) \widetilde{\varepsilon}_{j_{1}}^{[a]} \widetilde{\varepsilon}_{j_{2}}^{[a]}\right\}\right. \\
& \quad+\varphi^{2}\left\{\langle 3\rangle_{2^{2}} \widetilde{\Lambda}_{j_{1} j_{2}}^{(a, a)} \widetilde{\Lambda}_{j_{3} j_{4}}^{(a, a)}+(\varphi-1)\langle 6\rangle_{2^{1} \mid 1^{2}} \widetilde{\Lambda}_{j_{1} j_{2}}^{(a, a)} \widetilde{\varepsilon}_{j_{3}}^{a a]} \widetilde{\varepsilon}_{j_{4}}^{a a]}\right. \\
& \left.\left.\quad+(\varphi-1)^{2} \widetilde{\varepsilon}_{j_{1}}^{a]} \widetilde{\varepsilon}_{j_{2}}^{[a]} \widetilde{\varepsilon}_{j_{3}}^{a]} \widetilde{\varepsilon}_{j_{4}}^{a}\right\}\right] \\
= & \frac{\operatorname{ch}_{f}\left(t ; \omega^{2}\right)}{4}\left[-\left(\operatorname{tr}_{a}\right)^{2}-2 \varphi\left\{\operatorname{tr}_{a a}+(\varphi-1) \mathcal{E}_{a a}^{(2)}\right\}\right. \\
& \left.+\varphi^{2}\left\{2 \operatorname{tr}_{a a}+\left(\operatorname{tr}_{a}\right)^{2}+(\varphi-1)\left(4 \mathcal{E}_{a a}^{(2)}+2 \operatorname{tr}_{a} \mathcal{E}_{a}^{(2)}\right)+(\varphi-1)^{2}\left(\mathcal{E}_{a}^{(2)}\right)^{2}\right\}\right]
\end{aligned}
$$

Hence,

$$
\begin{aligned}
C_{n}(t)=\frac{1}{4} \sum_{a=1}^{q} \rho_{a}^{-2}[ & -\left(\operatorname{tr}_{a}\right)^{2}+2 \varphi\left(-\operatorname{tr}_{a a}+\mathcal{E}_{a a}^{(2)}\right) \\
& +\varphi^{2}\left\{2 \operatorname{tr}_{a a}+\left(\operatorname{tr}_{a}\right)^{2}-2\left(3 \mathcal{E}_{a a}^{(2)}+\operatorname{tr}_{a} \mathcal{E}_{a}^{(2)}\right)+\left(\mathcal{E}_{a}^{(2)}\right)^{2}\right\} \\
& \left.+2 \varphi^{3}\left\{2 \mathcal{E}_{a a}^{(2)}+\operatorname{tr}_{a} \mathcal{E}_{a}^{(2)}-\left(\mathcal{E}_{a}^{(2)}\right)^{2}\right\}+\varphi^{4}\left(\mathcal{E}_{a}^{(2)}\right)^{2}\right] \operatorname{ch}_{f}\left(t ; \omega^{2}\right)
\end{aligned}
$$

Using symmetricity of $\kappa_{j_{1}, j_{2}, j_{3}, j_{4}}^{(a)}$ under permutation, we have

$$
\begin{aligned}
\sum_{j_{1} j_{2} j_{3} j_{4}=1}^{p} \widetilde{\kappa}_{j_{1}, j_{2}, j_{3}, j_{4}}^{(a)} \widetilde{\Lambda}_{j_{1} j_{2}}^{(a, a)} Q_{j_{3} j_{4}}^{a a} & =\sum_{j_{1} j_{2} j_{3} j_{4}=1}^{p} \widetilde{\kappa}_{j_{1}, j_{2}, j_{3}, j_{4}}^{(a)} \widetilde{\Lambda}_{j_{3} j_{4}}^{(a, a)} Q_{j_{1} j_{2}}^{a a} \\
& =(\varphi-1) \kappa_{4}(a)+(\varphi-1)^{2} \kappa_{4}^{[2]}(a)
\end{aligned}
$$

and

$$
\begin{aligned}
& \sum_{j_{1} j_{2} j_{3} j_{4}=1}^{p} \widetilde{\kappa}_{j_{1}, j_{2}, j_{3}, j_{4}}^{(a)} Q_{j_{1} j_{2} j_{3} j_{4}}^{a \underset{a}{a} a} \\
& \quad=3(\varphi-1)^{2} \kappa_{4}(a)+6(\varphi-1)^{3} \kappa_{4}^{[2]}(a)+(\varphi-1)^{4} \kappa_{4}^{[4]}(a)
\end{aligned}
$$

Also, we have

$$
\begin{aligned}
\sum_{j_{1} j_{2} j_{3} j_{4}=1}^{p} \widetilde{\kappa}_{j_{1}, j_{2}, j_{3}, j_{4}}^{(a)} \widetilde{\Lambda}_{j_{1} j_{\ell}}^{(a, a)} \widetilde{\Lambda}_{j_{m} j_{n}}^{(a, a)} & =\kappa_{4}(a) \\
\text { for } \quad(\ell, m, n) & =(2,3,4),(3,2,4),(4,2,3)
\end{aligned}
$$

Then,

$$
C_{4,1}(t) \equiv \sum_{a=1}^{q} C_{4,1}^{(a)}(t)
$$




$$
\begin{aligned}
= & \frac{1}{8}\left[-K_{4}-2 \varphi\left\{K_{4}+(\varphi-1) K_{4}^{[2]}\right\}\right. \\
& \left.\quad+\varphi^{2}\left\{3 K_{4}+6(\varphi-1) K_{4}^{[2]}+(\varphi-1)^{2} K_{4}^{[4]}\right\}\right] \operatorname{ch}_{f}\left(t ; \omega^{2}\right), \\
C_{4,2}(t) \equiv & \sum_{a=1}^{q} C_{4,2}^{(a)}(t) \\
= & \frac{1}{4}\left[(\varphi-1) K_{4}+(\varphi-1)^{2} K_{4}^{[2]}\right. \\
& \left.\quad-\varphi\left\{3(\varphi-1) K_{4}+6(\varphi-1)^{2} K_{4}^{[2]}+(\varphi-1)^{3} K_{4}^{[4]}\right\}\right] \operatorname{ch}_{f}\left(t ; \omega^{2}\right), \\
C_{4,3}(t) \equiv & \sum_{a=1}^{q} C_{4,3}^{(a)}(t) \\
= & \frac{1}{24}\left\{3(\varphi-1)^{2} K_{4}+6(\varphi-1)^{3} K_{4}^{[2]}+(\varphi-1)^{4} K_{4}^{[4]}\right\} \operatorname{ch}_{f}\left(t ; \omega^{2}\right) .
\end{aligned}
$$

We finally evaluate

$$
C_{33}(t)=\left.\frac{\Xi_{0}}{2} \sum_{a a^{\prime}=1}^{q} \Xi_{1}^{(a)} \Xi_{1}^{\left(a^{\prime}\right)} e\left(\mathrm{i} t H_{0}\right)\right|_{\gamma^{*}=0, \Gamma^{*}=\Sigma^{*}}=\sum_{a a^{\prime}=1}^{q} C_{33}^{\left(a a^{\prime}\right)}(t),
$$

where

$$
C_{33}^{\left(a a^{\prime}\right)}(t)=\left.\frac{\Xi_{0}}{2} \Xi_{1}^{(a)} \Xi_{1}^{\left(a^{\prime}\right)} e\left(\mathrm{i} t H_{0}\right)\right|_{\gamma^{*}=0, \Gamma^{*}=\Sigma^{*}} .
$$

Since $C_{33}^{\left(a a^{\prime}\right)}(t)=C_{33}^{\left(a^{\prime} a\right)}(t)$, it is enough to consider

$$
C_{33}(t)=\sum_{j=1}^{3} C_{33, j}(t)
$$

with

$$
C_{33, j}(t)=\sum_{a=1}^{q} C_{33, j}^{(a a)}(t)+2 \sum_{a=1}^{q-1} \sum_{a^{\prime}=a+1}^{q} C_{33, j}^{\left(a a^{\prime}\right)}(t) \quad(j=1,2,3),
$$

where

$$
\begin{aligned}
C_{33,1}^{\left(a a^{\prime}\right)}(t)= & \frac{\Xi_{0}}{2} \sum_{j_{1} j_{2} j_{3} k_{4} k_{5} k_{6}=1}^{p} \widetilde{\kappa}_{j_{1}, j_{2}, j_{3}}^{(a)} \widetilde{\kappa}_{k_{4}, k_{5}, k_{6}}^{\left(a^{\prime}\right)} \partial_{j_{1}(a) j_{2}(a)}^{*} \partial_{j_{3}(a)}^{*} \partial_{k_{4}\left(a^{\prime}\right) k_{5}\left(a^{\prime}\right)}^{*} \partial_{k_{6}\left(a^{\prime}\right)}^{*} \\
& \times\left. e\left(\mathrm{i} t H_{0}\right)\right|_{\gamma^{*}=0, \Gamma^{*}=\Sigma^{*},}, \\
C_{33,2}^{\left(a a^{\prime}\right)}(t)= & \frac{\Xi_{0}}{12} \sum_{j_{1} j_{2} j_{3} k_{4} k_{5} k_{6}=1}^{p} \widetilde{\kappa}_{j_{1}, j_{2}, j_{3}}^{(a)} \widetilde{\kappa}_{k_{4}, k_{5}, k_{6}}^{\left(a^{\prime}\right)}\left(\partial_{j_{1}(a) j_{2}(a)}^{*} \partial_{j_{3}(a)}^{*} \partial_{k_{4}\left(a^{\prime}\right)}^{*} \partial_{k_{5}\left(a^{\prime}\right)}^{*} \partial_{k_{6}\left(a^{\prime}\right)}^{*}\right. \\
& \left.+\partial_{j_{1}(a)}^{*} \partial_{j_{2}(a)}^{*} \partial_{j_{3}(a)}^{*} \partial_{k_{4}\left(a^{\prime}\right) k_{5}\left(a^{\prime}\right)}^{*} \partial_{k_{6}\left(a^{\prime}\right)}^{*}\right)\left.e\left(\mathrm{i} t H_{0}\right)\right|_{\gamma^{*}=0, \Gamma^{*}=\Sigma^{*}}, \\
C_{33,3}^{\left(a a^{\prime}\right)}(t)= & \frac{\Xi_{0}}{72} \sum_{j_{1} j_{2} j_{3} k_{4} k_{5} k_{6}=1}^{p} \widetilde{\kappa}_{j_{1}, j_{2}, j_{3}}^{(a)} \widetilde{\kappa}_{k_{4}, k_{5}, k_{6}}^{\left(a^{\prime}\right)} \partial_{j_{1}(a)}^{*} \partial_{j_{2}(a)}^{*} \partial_{j_{3}(a)}^{*} \partial_{k_{4}\left(a^{\prime}\right)}^{*} \partial_{k_{5}\left(a^{\prime}\right)}^{*} \partial_{k_{6}\left(a^{\prime}\right)}^{*} \\
& \times\left. e\left(\mathrm{i} t H_{0}\right)\right|_{\gamma^{*}=0, \Gamma^{*}=\Sigma^{*}}
\end{aligned}
$$




$$
=\frac{\operatorname{ch}_{f}\left(t ; \omega^{2}\right)}{72} \sum_{j_{1} j_{2} j_{3} k_{4} k_{5} k_{6}=1}^{p} \widetilde{\kappa}_{j_{1}, j_{2}, j_{3}}^{(a)} \widetilde{\kappa}_{k_{4}, k_{5}, k_{6}}^{\left(a^{\prime}\right)} Q_{j_{1} j_{2} j_{3} k_{4} k_{5} k_{6}}^{a \underset{a_{2}}{a} a^{\prime} a^{\prime}}
$$

With regard to $C_{33,1}^{\left(a a^{\prime}\right)}(t)$ and $C_{33,2}^{\left(a a^{\prime}\right)}(t)$, we have

$$
\begin{aligned}
C_{33,1}^{\left(a a^{\prime}\right)}(t) & \\
= & \frac{\operatorname{ch}_{f}\left(t ; \omega^{2}\right)}{8} \sum_{j_{1} j_{2} j_{3} k_{4} k_{5} k_{6}=1}^{p} \widetilde{\kappa}_{j_{1}, j_{2}, j_{3}}^{(a)} \widetilde{\kappa}_{k_{4}, k_{5}, k_{6}}^{\left(a^{\prime}\right)} \\
& \times\left[\left(-2 \widetilde{\Lambda}_{j_{1} k_{4}}^{\left(a, a^{\prime}\right)} \widetilde{\Lambda}_{j_{2} k_{5}}^{\left(a, a^{\prime}\right)}+\widetilde{\Lambda}_{j_{1} j_{2}}^{(a, a)} \widetilde{\Lambda}_{k_{4} k_{5}}^{\left(a^{\prime}, a^{\prime}\right)}\right) Q_{j_{3} k_{6}}^{a a^{\prime}}\right. \\
& \left.\quad-\frac{\varphi}{\varphi-1}\left(\widetilde{\Lambda}_{k_{4} k_{5}}^{\left(a^{\prime}, a^{\prime}\right)} Q_{j_{1} j_{2} j_{3} k_{6}}^{a a a^{\prime}}+\widetilde{\Lambda}_{j_{1} j_{2}}^{(a, a)} Q_{j_{3} k_{4} k_{5} k_{6}}^{a a^{\prime} a^{\prime} a^{\prime}}\right)+\frac{\varphi^{2}}{(\varphi-1)^{2}} Q_{j_{1} j_{2} j_{3} k_{4} k_{5} k_{6}}^{a a a^{\prime} a^{\prime} a^{\prime}}\right]
\end{aligned}
$$

and

$$
\begin{aligned}
C_{33,2}^{\left(a a^{\prime}\right)}(t)= & \frac{\operatorname{ch}_{f}\left(t ; \omega^{2}\right)}{24} \sum_{j_{1} j_{2} j_{3} k_{4} k_{5} k_{6}=1}^{p} \widetilde{\kappa}_{j_{1}, j_{2}, j_{3}}^{(a)} \widetilde{\kappa}_{k_{4}, k_{5}, k_{6}}^{\left(a^{\prime}\right)} \\
& \times\left(\widetilde{\Lambda}_{k_{4} k_{5}}^{\left(a^{\prime}, a^{\prime}\right)} Q_{j_{1} j_{2} j_{3} k_{6}}^{a a a^{\prime}}+\widetilde{\Lambda}_{j_{1} j_{2}}^{(a, a)} Q_{j_{3} k_{4} k_{5} k_{6}}^{a a^{\prime} a^{\prime} a^{\prime}}-\frac{2 \varphi}{\varphi-1} Q_{j_{1} j_{2} j_{3} k_{4} k_{5} k_{6}}^{a a a^{\prime} a^{\prime} a^{\prime}}\right) .
\end{aligned}
$$

We can see that $C_{33}(t)$ depends on

$$
\begin{aligned}
& J_{1}=\sum_{a=1}^{q} \sum_{j_{1} j_{2} j_{3} k_{4} k_{5} k_{6}=1}^{p} \widetilde{\kappa}_{j_{1}, j_{2}, j_{3}}^{(a)} \widetilde{\kappa}_{k_{4}, k_{5}, k_{6}}^{(a)} \widetilde{\Lambda}_{j_{1} k_{4}}^{(a, a)} \widetilde{\Lambda}_{j_{2} k_{5}}^{(a, a)} Q_{j_{3} k_{6}}^{a a} \\
& +2 \sum_{a=1}^{q-1} \sum_{a^{\prime}=a+1}^{q} \sum_{j_{1} j_{2} j_{3} k_{4} k_{5} k_{6}=1}^{p} \widetilde{\kappa}_{j_{1}, j_{2}, j_{3}}^{(a)} \widetilde{\kappa}_{k_{4}, k_{5}, k_{6}}^{\left(a^{\prime}\right)} \widetilde{\Lambda}_{j_{1} k_{4}}^{\left(a, a^{\prime}\right)} \widetilde{\Lambda}_{j_{2} k_{5}}^{\left(a, a^{\prime}\right)} Q_{j_{3} k_{6}}^{a}, \\
& J_{2}=\sum_{a=1}^{q} \sum_{j_{1} j_{2} j_{3} k_{4} k_{5} k_{6}=1}^{p} \widetilde{\kappa}_{j_{1}, j_{2}, j_{3}}^{(a)} \widetilde{\kappa}_{k_{4}, k_{5}, k_{6}}^{(a)} \widetilde{\Lambda}_{j_{1} j_{2}}^{(a, a)} \widetilde{\Lambda}_{k_{4} k_{5}}^{(a, a)} Q_{j_{3} k_{6}}^{a a} \\
& +2 \sum_{a=1}^{q-1} \sum_{a^{\prime}=a+1}^{q} \sum_{j_{1} j_{2} j_{3} k_{4} k_{5} k_{6}=1}^{p} \widetilde{\kappa}_{j_{1}, j_{2}, j_{3}}^{(a)} \widetilde{\kappa}_{k_{4}, k_{5}, k_{6}}^{\left(a^{\prime}\right)} \widetilde{\Lambda}_{j_{1} j_{2}}^{(a, a)} \widetilde{\Lambda}_{k_{4} k_{5}}^{\left(a^{\prime}, a^{\prime}\right)} Q_{j_{3} k_{6}}^{a}, \\
& J_{3}=\sum_{a=1}^{q} \sum_{j_{1} j_{2} j_{3} k_{4} k_{5} k_{6}=1}^{p} \widetilde{\kappa}_{j_{1}, j_{2}, j_{3}}^{(a)} \widetilde{\kappa}_{k_{4}, k_{5}, k_{6}}^{(a)}\left(\widetilde{\Lambda}_{k_{4} k_{5}}^{(a, a)} Q_{j_{1} j_{2} j_{3} k_{6}}^{a \underset{a}{a} a}+\widetilde{\Lambda}_{j_{1} j_{2}}^{(a, a)} Q_{j_{3} k_{4} k_{5} k_{6}}^{a a a a}\right) \\
& +2 \sum_{a=1}^{q-1} \sum_{a^{\prime}=a+1}^{q} \sum_{j_{1} j_{2} j_{3} k_{4} k_{5}}^{p} \widetilde{\kappa}_{j_{6}=1}^{(a)}{ }_{j_{1}, j_{2}, j_{3}} \widetilde{\kappa}_{k_{4}, k_{5}, k_{6}}^{\left(a^{\prime}\right)} \\
& \times\left(\widetilde{\Lambda}_{k_{4} k_{5}}^{\left(a^{\prime}, a^{\prime}\right)} Q_{j_{1} j_{2} j_{3} k_{6}}^{a \text { a } a a^{\prime}}+\widetilde{\Lambda}_{j_{1} j_{2}}^{(a, a)} Q_{j_{3} k_{4} k_{5} k_{6}}^{a a^{\prime} a^{\prime} a^{\prime}}\right), \\
& J_{4}=\sum_{a=1}^{q} \sum_{j_{1} j_{2} j_{3} k_{4} k_{5} k_{6}=1}^{p} \widetilde{\kappa}_{j_{1}, j_{2}, j_{3}}^{(a)} \widetilde{\kappa}_{k_{4}, k_{5}, k_{6}}^{(a)} Q_{j_{1} j_{2} j_{3} k_{4} k_{5} k_{6}}^{a \underset{a}{a} a \underset{a}{a}}
\end{aligned}
$$




$$
+2 \sum_{a=1}^{q-1} \sum_{a^{\prime}=a+1}^{q} \sum_{j_{1} j_{2} j_{3} k_{4} k_{5} k_{6}=1}^{p} \widetilde{\kappa}_{j_{1}, j_{2}, j_{3}}^{(a)} \widetilde{\kappa}_{k_{4}, k_{5}, k_{6}}^{\left(a^{\prime}\right)} Q_{j_{1} j_{2} j_{3} k_{4} k_{5} k_{6}}^{a \underset{a}{a} a^{\prime} a^{\prime} a^{\prime}}
$$

Since $\kappa_{j_{1}, j_{2}, j_{3}}^{(a)} \kappa_{k_{1}, k_{2}, k_{3}}^{\left(a^{\prime}\right)}$ is not symmetric under index permutation, the evaluation of $J_{4}$ is most tedious. But, it is straightforward to see that for $1 \leq a \leq a^{\prime} \leq q$

$$
\begin{aligned}
& \sum_{j_{1} j_{2} j_{3} k_{4} k_{5} k_{6}=1}^{p} \widetilde{\kappa}_{j_{1}, j_{2}, j_{3}}^{(a)} \widetilde{\kappa}_{k_{4}, k_{5}, k_{6}}^{\left(a^{\prime}\right)} \widetilde{\Lambda}_{j_{1} k_{4}}^{\left(a, a^{\prime}\right)} \widetilde{\Lambda}_{j_{2} k_{5}}^{\left(a, a^{\prime}\right)} Q_{j_{3} k_{6}}^{a a^{\prime}} \\
& =(\varphi-1) \kappa_{33,1}\left(a, a^{\prime}\right)+(\varphi-1)^{2} \kappa_{33,1}^{[2]}\left(a, a^{\prime}\right), \\
& \sum_{j_{1} j_{2} j_{3} k_{4} k_{5} k_{6}=1}^{p} \widetilde{\kappa}_{j_{1}, j_{2}, j_{3}}^{(a)} \widetilde{\kappa}_{k_{4}, k_{5}, k_{6}}^{\left(a^{\prime}\right)} \widetilde{\Lambda}_{j_{1} j_{2}}^{(a, a)} \widetilde{\Lambda}_{k_{4} k_{5}}^{\left(a^{\prime}, a^{\prime}\right)} Q_{j_{3} k_{6}}^{a a^{\prime}} \\
& =(\varphi-1) \kappa_{33,2}\left(a, a^{\prime}\right)+(\varphi-1)^{2} \kappa_{3}^{[1]}(a) \kappa_{3}^{[1]}\left(a^{\prime}\right), \\
& \sum_{j_{1} j_{2} j_{3} k_{4} k_{5} k_{6}=1}^{p} \widetilde{\kappa}_{j_{1}, j_{2}, j_{3}}^{(a)} \widetilde{\kappa}_{k_{4}, k_{5}, k_{6}}^{\left(a^{\prime}\right)}\left(\widetilde{\Lambda}_{k_{4} k_{5}}^{\left(a^{\prime}, a^{\prime}\right)} Q_{j_{1} j_{2} j_{3} k_{6}}^{a a a^{\prime}}+\widetilde{\Lambda}_{j_{1} j_{2}}^{(a, a)} Q_{j_{3} k_{4} k_{5} k_{6}}^{a a^{\prime} a^{\prime} a^{\prime}}\right) \\
& =\sum_{j_{1} j_{2} j_{3} k_{4} k_{5} k_{6}=1}^{p} \widetilde{\kappa}_{j_{1}, j_{2}, j_{3}}^{(a)} \widetilde{\kappa}_{k_{4}, k_{5}, k_{6}}^{\left(a^{\prime}\right)} \widetilde{\Lambda}_{k_{4} k_{5}}^{\left(a^{\prime}, a^{\prime}\right)} \\
& \times\left[(\varphi-1)^{2}\left[3_{j_{1} j_{2}, j_{3}}\right] \widetilde{\Lambda}_{j_{1} j_{2}}^{(a, a)} \widetilde{\Lambda}_{j_{3} k_{6}}^{\left(a, a^{\prime}\right)}\right. \\
& +(\varphi-1)^{3}\left[3_{j_{1} j_{2}, j_{3}}\right]\left(\widetilde{\Lambda}_{j_{1} j_{2}}^{(a, a)} \widetilde{\varepsilon}_{j_{3}}^{[a]} \widetilde{\varepsilon}_{k_{6}}\left[a^{\prime}\right]+\widetilde{\varepsilon}_{j_{1}}^{[a]} \widetilde{\varepsilon}_{j_{2}}^{[a]} \widetilde{\Lambda}_{j_{3} k_{6}}^{\left(a, a^{\prime}\right)}\right) \\
& \left.+(\varphi-1)^{4} \widetilde{\varepsilon}_{j_{1}}^{[a]} \widetilde{\varepsilon}_{j_{2}}^{[a]} \widetilde{\varepsilon}_{j_{3}}^{[a]} \widetilde{\varepsilon}_{k_{6}}^{\left[a^{\prime}\right]}\right] \\
& +\sum_{j_{1} j_{2} j_{3} k_{4} k_{5} k_{6}=1}^{p} \widetilde{\kappa}_{j_{1}, j_{2}, j_{3}}^{(a)} \widetilde{\kappa}_{k_{4}, k_{5}, k_{6}}^{\left(a^{\prime}\right)} \widetilde{\Lambda}_{j_{1} j_{2}}^{(a, a)} \\
& \times\left[(\varphi-1)^{2}\left[3_{k_{4} k_{5}, k_{6}}\right] \widetilde{\Lambda}_{k_{4} k_{5}}^{\left(a^{\prime}, a^{\prime}\right)} \widetilde{\Lambda}_{j_{3} k_{6}}^{\left(a, a^{\prime}\right)}\right. \\
& +(\varphi-1)^{3}\left[3_{k_{4} k_{5}, k_{6}}\right]\left(\widetilde{\varepsilon}_{j_{3}}^{[a]} \widetilde{\Lambda}_{k_{4} k_{5}}^{\left(a^{\prime}, a^{\prime}\right)} \widetilde{\varepsilon}_{k_{6}}^{\left[a^{\prime}\right]}+\widetilde{\Lambda}_{j_{3} k_{6}}^{\left(a, a^{\prime}\right)} \widetilde{\varepsilon}_{k_{4}}^{\left.a^{\prime}\right]} \widetilde{\varepsilon}_{k_{5}}^{\left.a^{\prime}\right]}\right) \\
& \left.+(\varphi-1)^{4} \widetilde{\varepsilon}_{j_{3}}^{[a]} \widetilde{\varepsilon}_{k_{4}}^{\left[a^{\prime}\right]} \widetilde{\varepsilon}_{k_{5}}^{\left[a^{\prime}\right]} \widetilde{\varepsilon}_{k_{6}}^{\left[a^{\prime}\right]}\right] \\
& =6(\varphi-1)^{2} \kappa_{33,2}\left(a, a^{\prime}\right)+6(\varphi-1)^{3} \kappa_{3}^{[1]}(a) \kappa_{3}^{[1]}\left(a^{\prime}\right) \\
& +3(\varphi-1)^{3}\left\{\kappa_{32,2}^{[2]}\left(a^{\prime}, a\right)+\kappa_{32,2}^{[2]}\left(a, a^{\prime}\right)\right\} \\
& +(\varphi-1)^{4}\left\{\kappa_{3}^{[1]}\left(a^{\prime}\right) \kappa_{3}^{[3]}(a)+\kappa_{3}^{[1]}(a) \kappa_{3}^{[3]}\left(a^{\prime}\right)\right\}
\end{aligned}
$$

and

$$
\begin{gathered}
\sum_{j_{1} j_{2} j_{3} k_{4} k_{5} k_{6}=1}^{p} \widetilde{\kappa}_{j_{1}, j_{2}, j_{3}}^{(a)} \widetilde{\kappa}_{k_{4}, k_{5}, k_{6}}^{\left(a^{\prime}\right)} Q_{j_{1} j_{2} j_{3} k_{4} k_{5} k_{6}}^{a \text { a } a^{\prime} a^{\prime} a^{\prime}} \\
=\sum_{j_{1} j_{2} j_{3} k_{4} k_{5} k_{6}=1}^{p} \widetilde{\kappa}_{j_{1}, j_{2}, j_{3}}^{(a)} \widetilde{\kappa}_{k_{4}, k_{5}, k_{6}}^{\left(a^{\prime}\right)}
\end{gathered}
$$




$$
\begin{aligned}
& \times\left[( \varphi - 1 ) ^ { 3 } \left(\left[6_{j_{1}, j_{2}, j_{3}}\right] \widetilde{\Lambda}_{j_{1} k_{4}}^{\left(a, a^{\prime}\right)} \widetilde{\Lambda}_{j_{2} k_{5}}^{\left(a, a^{\prime}\right)} \widetilde{\Lambda}_{j_{3} k_{6}}^{\left(a, a^{\prime}\right)}\right.\right. \\
& \left.+\left[3_{j_{1} j_{2}, j_{3}}\right]\left[3_{k_{4}, k_{5} k_{6}}\right] \widetilde{\Lambda}_{j_{1} j_{2}}^{(a, a)} \widetilde{\Lambda}_{j_{3} k_{4}}^{\left(a, a^{\prime}\right)} \widetilde{\Lambda}_{k_{5} k_{6}}^{\left(a^{\prime}, a^{\prime}\right)}\right) \\
& +(\varphi-1)^{4}\left(\left[3_{j_{1} j_{2}, j_{3}}\right]\left[3_{k_{4} k_{5}, k_{6}}\right] \widetilde{\Lambda}_{j_{1} j_{2}}^{(a, a)} \widetilde{\varepsilon}_{j_{3}}^{[a]} \widetilde{\Lambda}_{k_{4} k_{5}}^{\left(a^{\prime}, a^{\prime}\right)} \widetilde{\varepsilon}_{k_{6}}^{\left[a^{\prime}\right]}\right. \\
& +\left[3_{j_{1} j_{2}, j_{3}}\right]\left[6_{k_{4}, k_{5}, k_{6}}\right] \widetilde{\Lambda}_{j_{1} k_{4}}^{\left(a, a^{\prime}\right)} \widetilde{\Lambda}_{j_{2} k_{5}}^{\left(a, a^{\prime}\right)} \widetilde{\varepsilon}_{j_{3}}^{[a]} \widetilde{\varepsilon}_{k_{6}}^{\left[a^{\prime}\right]} \\
& +\left[3_{j_{1}, j_{2} j_{3}}\right]\left[3_{k_{4}, k_{5} k_{6}}\right] \widetilde{\Lambda}_{j_{1} k_{4}}^{\left(a, a^{\prime}\right)} \widetilde{\Lambda}_{k_{5} k_{6}}^{\left(a^{\prime}, a^{\prime}\right)} \widetilde{\varepsilon}_{j_{2}}^{[a]} \widetilde{\varepsilon}_{j_{3}}^{[a]} \\
& +\left[3_{k_{4}, k_{5} k_{6}}\right]\left[3_{j_{1}, j_{2} j_{3}}\right] \widetilde{\Lambda}_{j_{1} k_{4}}^{\left(a, a^{\prime}\right)} \widetilde{\Lambda}_{j_{2} j_{3}}^{(a, a)} \widetilde{\varepsilon}_{k_{5}}\left[a^{\prime}\right] \widetilde{\varepsilon}_{k_{6}}\left[a^{\prime}\right] \\
& +(\varphi-1)^{5}\left(\left[3_{j_{1} j_{2}, j_{3}}\right] \widetilde{\Lambda}_{j_{1} j_{2}}^{(a, a)} \widetilde{\varepsilon}_{j_{3}}^{a a} \widetilde{\varepsilon}_{k_{4}}^{\left[a^{\prime}\right]} \widetilde{\varepsilon}_{k_{5}}^{\left[a^{\prime}\right]} \widetilde{\varepsilon}_{k_{6}}^{\left.a^{\prime}\right]}\right. \\
& +\left[3_{k_{4} k_{5}, k_{6}}\right] \widetilde{\varepsilon}_{j_{1}}^{[a]} \widetilde{\varepsilon}_{j_{2}}^{[a]} \widetilde{\varepsilon}_{j_{3}}^{[a]} \widetilde{\Lambda}_{k_{4} k_{5}}^{\left(a^{\prime}, a^{\prime}\right)} \widetilde{\varepsilon}_{k_{6}}^{\left[a^{\prime}\right]} \\
& \left.+\left[3_{j_{1}, j_{2} j_{3}}\right]\left[3_{k_{4}, k_{5} k_{6}}\right] \widetilde{\Lambda}_{j_{1} k_{4}}^{\left(a, a^{\prime}\right)} \widetilde{\varepsilon}_{j_{2}}^{[a]} \widetilde{\varepsilon}_{j_{3}}^{[a]} \widetilde{\varepsilon}_{k_{5}}^{\left[a^{\prime}\right]} \widetilde{\varepsilon}_{k_{6}}^{\left[a^{\prime}\right]}\right) \\
& \left.+(\varphi-1)^{6} \widetilde{\varepsilon}_{j_{1}}^{[a]} \widetilde{\varepsilon}_{j_{2}}^{[a]} \widetilde{\varepsilon}_{j_{3}}^{[a]} \widetilde{\varepsilon}_{k_{4}}^{\left[a^{\prime}\right]} \widetilde{\varepsilon}_{k_{5}}^{\left[a^{\prime}\right]} \widetilde{\varepsilon}_{k_{6}}^{\left[a^{\prime}\right]}\right] \\
& =3(\varphi-1)^{3}\left\{2 \kappa_{33,1}\left(a, a^{\prime}\right)+3 \kappa_{33,2}\left(a, a^{\prime}\right)\right\} \\
& +9(\varphi-1)^{4}\left\{\kappa_{3}^{[1]}(a) \kappa_{3}^{[1]}\left(a^{\prime}\right)+2 \kappa_{33,1}^{[2]}\left(a, a^{\prime}\right)+\kappa_{33,2}^{[2]}\left(a^{\prime}, a\right)+\kappa_{33,2}^{[2]}\left(a, a^{\prime}\right)\right\} \\
& +3(\varphi-1)^{5}\left\{\kappa_{3}^{[1]}(a) \kappa_{3}^{[3]}\left(a^{\prime}\right)+\kappa_{3}^{[1]}\left(a^{\prime}\right) \kappa_{3}^{[3]}(a)+3 \kappa_{33}^{[4]}\left(a, a^{\prime}\right)\right\} \\
& +(\varphi-1)^{6} \kappa_{3}^{[3]}(a) \kappa_{3}^{[3]}\left(a^{\prime}\right) \text {. }
\end{aligned}
$$

Further, in view of the definition, we note the symmetricity of $\kappa_{33,1}\left(a, a^{\prime}\right)=$ $\kappa_{33,1}\left(a^{\prime}, a\right), \kappa_{33,2}\left(a, a^{\prime}\right)=\kappa_{33,2}\left(a^{\prime}, a\right), \kappa_{33,1}^{[2]}\left(a, a^{\prime}\right)=\kappa_{33,1}^{[2]}\left(a^{\prime}, a\right)$ and $\kappa_{33}^{[4]}\left(a, a^{\prime}\right)=$ $\kappa_{33}^{[4]}\left(a^{\prime}, a\right)$, since $\widetilde{\Lambda}_{j k}^{\left(a, a^{\prime}\right)}$ is the $\left(j(a), k\left(a^{\prime}\right)\right)$-th element of $p q \times p q$ symmetric matrix $\widetilde{\Lambda}$ given in Remark 2 , that is,

$$
\widetilde{\Lambda}_{j k}^{\left(a, a^{\prime}\right)}=[\widetilde{\Lambda}]_{j(a), k\left(a^{\prime}\right)}=[\widetilde{\Lambda}]_{k\left(a^{\prime}\right), j(a)}=\widetilde{\Lambda}_{k j}^{\left(a^{\prime}, a\right)} .
$$

We then have

$$
\begin{aligned}
J_{1}= & (\varphi-1) K_{33,1}+(\varphi-1)^{2} K_{33,1}^{[2]}, \\
J_{2}= & (\varphi-1) K_{33,2}+(\varphi-1)^{2}\left(K_{3}^{[1]}\right)^{2}, \\
\frac{J_{3}}{2}= & 3(\varphi-1)^{2} K_{33,2}+3(\varphi-1)^{3}\left\{\left(K_{3}^{[1]}\right)^{2}+K_{33,2}^{[2]}\right\}+(\varphi-1)^{4} K_{3}^{[1]} K_{3}^{[3]}, \\
J_{4}= & 3(\varphi-1)^{3}\left(2 K_{33,1}+3 K_{33,2}\right)+9(\varphi-1)^{4}\left\{\left(K_{3}^{[1]}\right)^{2}+2\left(K_{33,1}^{[2]}+K_{33,2}^{[2]}\right)\right\} \\
& +3(\varphi-1)^{5}\left(2 K_{3}^{[1]} K_{3}^{[3]}+3 K_{33}^{[4]}\right)+(\varphi-1)^{6}\left(K_{3}^{[3]}\right)^{2} .
\end{aligned}
$$

It follows that

$$
\begin{aligned}
C_{33,1}(t)= & \frac{1}{8}\left\{-2 J_{1}+J_{2}-\frac{\varphi}{\varphi-1} J_{3}+\frac{\varphi^{2}}{(\varphi-1)^{2}} J_{4}\right\} \operatorname{ch}_{f}\left(t ; \omega^{2}\right) \\
=\frac{1}{8} & -2\left\{(\varphi-1) K_{33,1}+(\varphi-1)^{2} K_{33,1}^{[2]}\right\}+(\varphi-1) K_{33,2}+(\varphi-1)^{2}\left(K_{3}^{[1]}\right)^{2} \\
& -2 \varphi\left[3(\varphi-1) K_{33,2}+3(\varphi-1)^{2}\left\{\left(K_{3}^{[1]}\right)^{2}+K_{33,2}^{[2]}\right\}\right.
\end{aligned}
$$




$$
\begin{aligned}
&+\left.(\varphi-1)^{3} K_{3}^{[1]} K_{3}^{[3]}\right] \\
&+ \varphi^{2}\left[3(\varphi-1)\left(2 K_{33,1}+3 K_{33,2}\right)\right. \\
&+ 9(\varphi-1)^{2}\left\{\left(K_{3}^{[1]}\right)^{2}+2\left(K_{33,1}^{[2]}+K_{33,2}^{[2]}\right)\right\} \\
&\left.\left.+3(\varphi-1)^{3}\left(2 K_{3}^{[1]} K_{3}^{[3]}+3 K_{33}^{[4]}\right)+(\varphi-1)^{4}\left(K_{3}^{[3]}\right)^{2}\right]\right] \operatorname{ch}_{f}\left(t ; \omega^{2}\right), \\
& C_{33,2}(t)=\frac{1}{24}\left(J_{3}-\frac{2 \varphi}{\varphi-1} J_{4}\right) \operatorname{ch}_{f}\left(t ; \omega^{2}\right) \\
&=\frac{1}{12}\left[3(\varphi-1)^{2} K_{33,2}+3(\varphi-1)^{3}\left\{\left(K_{3}^{[1]}\right)^{2}+K_{33,2}^{[2]}\right\}+(\varphi-1)^{4} K_{3}^{[1]} K_{3}^{[3]}\right. \\
& \quad-\varphi\left[3(\varphi-1)^{2}\left(2 K_{33,1}+3 K_{33,2}\right)\right. \\
&+9(\varphi-1)^{3}\left\{\left(K_{3}^{[1]}\right)^{2}+2\left(K_{33,1}^{[2]}+K_{33,2}^{[2]}\right)\right\} \\
&\left.\left.+3(\varphi-1)^{4}\left(2 K_{3}^{[1]} K_{3}^{[3]}+3 K_{33}^{[4]}\right)+(\varphi-1)^{5}\left(K_{3}^{[3]}\right)^{2}\right]\right] \operatorname{ch}_{f}\left(t ; \omega^{2}\right), \\
& C_{33,3}(t)=\frac{J_{4}}{72} \operatorname{ch}_{f}\left(t ; \omega^{2}\right) \\
& \frac{1}{72}\left[3(\varphi-1)^{3}\left(2 K_{33,1}+3 K_{33,2}\right)\right. \\
&+9(\varphi-1)^{4}\left\{\left(K_{3}^{[1]}\right)^{2}+2\left(K_{33,1}^{[2]}+K_{33,2}^{[2]}\right)\right\} \\
&\left.+3(\varphi-1)^{5}\left(2 K_{3}^{[1]} K_{3}^{[3]}+3 K_{33}^{[4]}\right)+(\varphi-1)^{6}\left(K_{3}^{[3]}\right)^{2}\right] \operatorname{ch}_{f}\left(t ; \omega^{2}\right) .
\end{aligned}
$$

After long but straightforward simplifications for collecting terms of $\varphi^{\ell}$, we obtain

$$
C_{n}(t)+\sum_{j=1}^{3}\left\{C_{4, j}(t)+C_{33, j}(t)\right\}=\sum_{\ell=0}^{6} \pi_{2, \ell} \varphi^{\ell} \operatorname{ch}_{f}\left(t ; \omega^{2}\right)
$$

Here, it may be noted that $\sum_{j=1}^{3}\left\{C_{4, j}(t)+C_{33, j}(t)\right\}$ has the same form as Hotelling's one-sample $T^{2}$ (see Kakizawa and Iwashita (2005)).

Appendix B: Evaluation of (4.4)

It is easy to see that

$$
\begin{gathered}
\left.\left\{\frac{\Xi_{1}}{N^{1 / 2}}+\frac{1}{N}\left(\Xi_{n}+\Xi_{2}+\frac{1}{2} \Xi_{1}^{2}\right)\right\} \exp \left\{\mathrm{i} t H_{0}\left(\gamma^{*}+\varepsilon^{*}, \Gamma^{*}\right)\right\}\right|_{\Gamma^{*}=\Sigma^{*}} \\
=\left\{\frac{P_{1}\left(\gamma^{*}+\varepsilon^{*} ; \mathrm{i} t\right)}{N^{1 / 2}}+\frac{P_{2}\left(\gamma^{*}+\varepsilon^{*} ; \mathrm{i} t\right)}{N}\right\} \exp \left\{\mathrm{i} t\left(\gamma^{*}+\varepsilon^{*}\right)^{\prime}\left(\Sigma^{*}\right)^{-1}\left(\boldsymbol{\gamma}^{*}+\varepsilon^{*}\right)\right\}
\end{gathered}
$$

where $P_{1}\left(\gamma^{*} ; \alpha\right)$ and $P_{2}\left(\gamma^{*} ; \alpha\right)$ are polynomials of $\alpha \in C$ and $\gamma^{*}=\left(\gamma_{j}^{*}\right)$ whose coefficients do not depend on $N$. Furthermore, we know

$$
\begin{aligned}
&\left\{\frac{\Xi_{1}}{N^{1 / 2}}+\right.\left.\frac{1}{N}\left(\Xi_{n}+\Xi_{2}+\frac{1}{2} \Xi_{1}^{2}\right)\right\} \exp \left\{\mathrm{i} t H\left(\gamma^{*}+\varepsilon^{*}, \Gamma^{*}\right)\right\} \mid \Gamma^{*}=\Sigma^{*} \\
&-\left\{\frac{P_{1}\left(\gamma^{*}+\varepsilon^{*} ; \mathrm{i} t\right)}{N^{1 / 2}}+\frac{P_{2}\left(\gamma^{*}+\varepsilon^{*} ; \mathrm{i} t\right)}{N}\right\} \exp \left\{\mathrm{i} t H\left(\gamma^{*}+\varepsilon^{*}, \Sigma^{*}\right)\right\} \\
&=R_{N}\left(\gamma^{*}+\varepsilon^{*} ; \mathrm{i} t\right) \exp \left\{\mathrm{i} t H\left(\gamma^{*}+\varepsilon^{*}, \Sigma^{*}\right)\right\},
\end{aligned}
$$


where $R_{N}\left(\gamma^{*} ; \alpha\right)$ is a polynomial of $\alpha \in C$ and $\gamma^{*}=\left(\gamma_{j}^{*}\right)$ whose coefficients depend on $N^{-3 / 2}, \ldots, N^{-13}$. As discussed in Kakizawa and Iwashita (2005), operating $\Xi_{0}$ to an analytic function $F\left(\gamma^{*}+\varepsilon^{*}\right)$ yields the expectation of $F(\boldsymbol{X})$ with respect to the normal distribution $\boldsymbol{X} \sim N_{f}\left(\varepsilon^{*}, \Sigma^{*}\right)$. Specializing

$$
\begin{aligned}
F(\boldsymbol{X})= & \left\{1+\frac{P_{1}(\boldsymbol{X} ; \mathrm{i} t)}{N^{1 / 2}}+\frac{P_{2}(\boldsymbol{X} ; \mathrm{i} t)}{N}+R_{N}(\boldsymbol{X} ; \mathrm{i} t)\right\} \exp \left\{\mathrm{i} t H\left(\boldsymbol{X}, \Sigma^{*}\right)\right\} \\
& \text { or } \quad\left\{1+\frac{P_{1}(\boldsymbol{X} ; \mathrm{i} t)}{N^{1 / 2}}+\frac{P_{2}(\boldsymbol{X} ; \mathrm{i} t)}{N}\right\} \exp \left\{\mathrm{i} t H_{0}\left(\boldsymbol{X}, \Sigma^{*}\right)\right\},
\end{aligned}
$$

we have

$$
\begin{aligned}
& \Xi_{0}\{1+\left.\frac{\Xi_{1}}{N^{1 / 2}}+\frac{1}{N}\left(\Xi_{n}+\Xi_{2}+\frac{1}{2} \Xi_{1}^{2}\right)\right\} \\
& \times\left.\exp \left\{\mathrm{i} t H\left(\boldsymbol{\gamma}^{*}+\varepsilon^{*}, \Gamma^{*}\right)\right\}\right|_{\gamma^{*}=0, \Gamma^{*}=\Sigma^{*}} \\
&=E_{X}\left\{1+\frac{P_{1}(\boldsymbol{X} ; \mathrm{i} t)}{N^{1 / 2}}+\frac{P_{2}(\boldsymbol{X} ; \mathrm{i} t)}{N}+R_{N}(\boldsymbol{X} ; \mathrm{i} t)\right\} \\
& \quad\left.\times \exp \left\{\mathrm{i} t \boldsymbol{X}^{\prime}\left(\Sigma^{*}\right)^{-1} \boldsymbol{X}-\frac{2 \mathrm{i} t}{N} H_{1}\left(\boldsymbol{X}, \Sigma^{*}\right)+\frac{\mathrm{i} t}{N^{2}} H_{2}\left(\boldsymbol{X}, \Sigma^{*}\right)\right\}\right] \\
&=E_{X}[\left\{1+\frac{P_{1}(\boldsymbol{X} ; \mathrm{i} t)}{N^{1 / 2}}+\frac{P_{2}(\boldsymbol{X} ; \mathrm{i} t)}{N}-\frac{2 \mathrm{i} t H_{1}\left(\boldsymbol{X}, \Sigma^{*}\right)}{N}\right\} \\
&\left.\quad \times \exp \left\{\mathrm{i} t \boldsymbol{X}^{\prime}\left(\Sigma^{*}\right)^{-1} \boldsymbol{X}\right\}\right] \\
&+\mathrm{o}\left(N^{-1}\right)
\end{aligned}
$$

and

$$
\begin{aligned}
E_{X}[ & \left.\left\{1+\frac{P_{1}(\boldsymbol{X} ; \mathrm{i} t)}{N^{1 / 2}}+\frac{P_{2}(\boldsymbol{X} ; \mathrm{i} t)}{N}\right\} \exp \left\{\mathrm{it} \boldsymbol{X}^{\prime}\left(\Sigma^{*}\right)^{-1} \boldsymbol{X}\right\}\right] \\
= & \Xi_{0}\left\{1+\frac{\Xi_{1}}{N^{1 / 2}}+\frac{1}{N}\left(\Xi_{n}+\Xi_{2}+\frac{1}{2} \Xi_{1}^{2}\right)\right\} \\
& \times\left.\exp \left\{\mathrm{it} H_{0}\left(\boldsymbol{\gamma}^{*}+\varepsilon^{*}, \Gamma^{*}\right)\right\}\right|_{\gamma^{*}=0, \Gamma^{*}=\Sigma^{*}} \\
= & \operatorname{ch}_{f}\left(t ; \omega^{2}\right)\left(1+\sum_{r=1}^{2} \frac{1}{N^{r / 2}} \sum_{\ell=0}^{3 r} \pi_{r, \ell} \varphi^{\ell}\right)
\end{aligned}
$$

(see Appendix A). It remains to calculate the integral

$$
\begin{aligned}
E_{X}[ & \left.H_{1}\left(\boldsymbol{X}, \Sigma^{*}\right) \exp \left\{\mathrm{it} \boldsymbol{X}^{\prime}\left(\Sigma^{*}\right)^{-1} \boldsymbol{X}\right\}\right] \\
& =\sum_{j=1}^{3} c_{j}(-\mathrm{i})^{j}\left(\frac{d}{d t}\right)^{j} E_{X}\left[\exp \left\{\mathrm{it} \boldsymbol{X}^{\prime}\left(\Sigma^{*}\right)^{-1} \boldsymbol{X}\right\}\right] \\
& =\sum_{j=1}^{3} c_{j}(-\mathrm{i})^{j}\left(\frac{d}{d t}\right)^{j} \frac{1}{(1-2 \mathrm{i} t)^{f / 2}} \exp \left\{\frac{\omega^{2}}{2(1-2 \mathrm{i} t)}-\frac{\omega^{2}}{2}\right\}
\end{aligned}
$$




$$
\begin{aligned}
=\operatorname{ch}_{f}\left(t ; \omega^{2}\right)[ & c_{1}\left(\varphi f+\varphi^{2} \omega^{2}\right)+c_{2}\left\{\varphi^{2} f(f+2)\right. \\
& \left.+2 \varphi^{3}(f+2) \omega^{2}+\varphi^{4} \omega^{4}\right\} \\
& +c_{3}\left\{\varphi^{3} f(f+2)(f+4)+3 \varphi^{4}(f+2)(f+4) \omega^{2}\right. \\
& \left.\left.+3 \varphi^{5}(f+4) \omega^{4}+\varphi^{6} \omega^{6}\right\}\right] .
\end{aligned}
$$

Multiplying (B.3) by $-2 \mathrm{i} t=(1-\varphi) / \varphi$, (4.4) follows from (B.1) and (B.2).

\section{REFERENCES}

Bhattacharya, R. and Denker, M. (1990). Asymptotic Statistics, Birkhäuser-Verlag, Basel.

Bhattacharya, R. N. and Ghosh, J. K. (1978). On the validity of the formal Edgeworth expansion, Ann. Statist., 6, 434-451. Correction: (1980). 8, 1399.

Bhattacharya, R. N. and Rao, R. R. (1976). Normal Approximation and Asymptotic Expansions, Wiley, New York.

Chandra, T. K. and Ghosh, J. K. (1980). Valid asymptotic expansions for the likelihood ratio and other statistics under contiguous alternatives, Sankhya $, \mathbf{4 2}, 170-184$.

Chibisov, D. M. (1972). An asymptotic expansion for the distribution of a statistic admitting an asymptotic expansion, Theory Prob. Appl., 17, 620-630.

Cordeiro, G. M. and Ferrari, S. L. P. (1991). A modified score test statistic having chi-squared distribution to order $n^{-1}$, Biometrika, 78, 573-582.

Eaton, M. L. and Perlman, M. D. (1973). The non-singularity of generalized sample covariance matrices, Ann. Statist., 1, 710-717.

Fujikoshi, Y. (1997). An asymptotic expansion for the distribution of Hotelling's $T^{2}$-statistic under nonnormality, J. Mult. Anal., 61, 187-193.

Fujikoshi, Y. (2002a). Asymptotic expansions for the distributions of multivariate basic statistics and one-way MANOVA tests under nonnormality, J. Statist. Plan. Inf., 108, 263-282.

Fujikoshi, Y. (2002b). Some recent results on asymptotic expansions of multivariate test statistics for mean vectors under nonnormality, Calcutta Statist. Assoc. Bulletin, 52, 1-46.

Gupta, A. K., Xu, J. and Fujikoshi, Y. (2006). An asymptotic expansion of the distribution of Rao's $U$-statistic under a general condition, J. Mult. Anal., 97, 492-513.

Hall, P. (1992). The Bootstrap and Edgeworth Expansion, Springer, New York.

Ito, K. (1969). On the effect of heteroscedasticity and nonnormality upon some multivariate test procedures, Multivariate Analysis II (ed. P. R. Krishnaiah), pp. 87-120, Academic Press, New York.

James, G. S. (1954). Tests of linear hypotheses in univariate and multivariate analysis when the ratios of the population variances are unknown, Biometrika, 41, 19-43.

Kakizawa, Y. (1996). Higher order monotone Bartlett-type adjustment for some multivariate test statistics, Biometrika, 83, 923-927.

Kakizawa, Y. (2005). A comparison of local powers of a class of tests for multivariate linear hypothesis under general distributions. Discussion Paper Series A: No. 2005-142 \& 162, 2006-168 and 2007-188, Faculty of Economics, Hokkaido University.

Kakizawa, Y. (2006). Siotani's modified second approximation for multiple comparisons of mean vectors, SUT Journal of Mathematics, 42, 59-96.

Kakizawa, Y. and Iwashita, T. (2005). Hotelling's one-sample and two-sample $T^{2}$ tests and the multivariate Behrens-Fisher problem under nonnormality (this paper was accepted for publication in April 30, 2006), J. Statist. Plan. Inf. (to appear).

Kakizawa, Y. and Iwashita, T. (2008). A comparison of higher-order local powers of a class of one-way MANOVA tests under general distributions, doi:10.1016/j.jmva.2007.07.005, J. Mult. Anal. (to appear).

Kano, Y. (1995). An asymptotic expansion of the distribution of Hotelling's $T^{2}$-statistic under general distributions, Amer. J. Math. Management Sciences, 15, 317-341.

Magdalinos, M. A. (1992). Stochastic expansions and asymptotic approximations, Econometric Theory, 8, 343-367. 
Wakaki, H., Yanagihara, H. and Fujikoshi, Y. (2002). Asymptotic expansions of the null distributions of test statistics for multivariate linear hypothesis under nonnormality, Hiroshima Math. J., 32, 17-50.

Yanagihara, H. (2000). Asymptotic expansion of the null distribution of one-way anova test statistic for heteroscedastic case under nonnormality, Commun. Statist. Theory Meth., 29, 463-476.

Yanagihara, H. (2001). Asymptotic expansion of the null distribution of three test statistics in a nonnormal GMANOVA model, Hiroshima Math. J., 31, 213-262. 